\title{
The Turbulent Boundary Layer in a Compressible Fluid
}

\author{
Donald Coles \\ The Rand Corporation, Santa Monica, California \\ (Received 22 October 1963; final mamuscript received 22 April 1964)
}

\begin{abstract}
A transformation is derived from first principles to reduce the boundary-layer equations for a general compressible two-dimensional flow to incompressible form. For the case of boundary-layer flow of a Newtonian fluid past a smooth wall, but with no other restrictions, it is shown that the combination $\left(\rho_{\circ} \mu_{x_{0}} / \rho_{\mathrm{w}} \mu_{\mathrm{w}}\right) C_{f} \operatorname{Re}_{\theta}$ is an invariant of the transformation. This result is called the law of corresponding stations. In order to apply the transformation to the problem of the turbulent boundary layer on a smooth wall, it is assumed that the sublayer Reynolds number is unaffected by compressibility or heat transfer provided the density and viscosity are evaluated at a mean sublayer temperature defined by the transformation. Explicit formulas are obtained for the effect of Mach number and heat transfer on surface friction when the fluid is a perfect gas, the pressure is constant, and the stagnation temperature is constant or linear in the velocity. An appendix contains a brief critical discussion of the mean-temperature hypothesis, the laminar-film hypothesis, and other analytical ideas related to the idea of a transformation.
\end{abstract}

\section{INTRODUCTION}

\section{Outline and Prospectus}

$\mathrm{T}$ HIS paper is concerned primarily with the effect of compressibility on turbulent skin friction in adiabatic flow at constant pressure. It has become a tradition in the literature concerning this problem to present the final result of both analytical and experimental work in the manner of Fig. 1, where the ratio of compressible to incompressible friction coefficient is plotted against Mach number. This paper, however, inadvertently follows another and stronger tradition. This is that research in turbulence is most respectable when it achieves the reduction of a given set of empirical data to a straight lineand hence to one or two empirical constants-in suitably chosen coordinates. That the problem at hand can be treated in this way is illustrated schematically by the evolution of Fig. 1 into Fig. 2 and then into Fig. 3. In fact, a description of the coordinate changes involved in going from one figure to the next serves both as an outline and as a summary of the analysis to follow.

The real starting point of the analysis is not Fig. 1 itself, but a collateral problem that arises

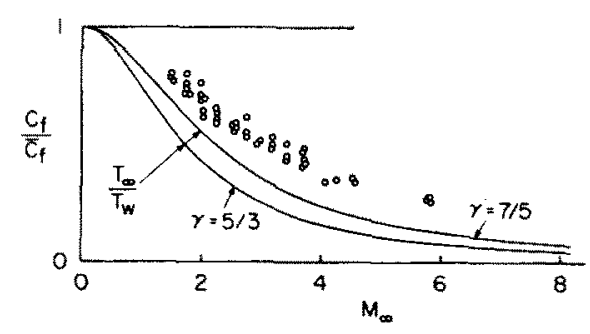

FrG. 1. Floating-element measurements of local surface friction in air.

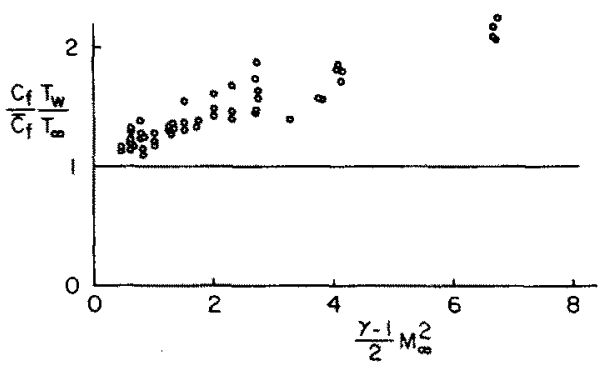

Frg. 2. Data of Fig. 1 normalized with respect to temperature ratio.

in the preparation of this figure from empirical data. This problem is to specify some condition connecting the local friction coefficients $C_{\mathrm{f}}$ and $\bar{C}_{\mathrm{f}}$. Most authors agree that these coefficients should be evaluated at the same Reynolds number, although they do not agree on the particular variables, especially the length, that should make up this Reynolds number. If any exit from this difficulty exists, it is almost certainly through the use of a transformation representing a genuine kinematic and dynamic correspondence between the two flows. In Sees. I through III, therefore, such a transformation is

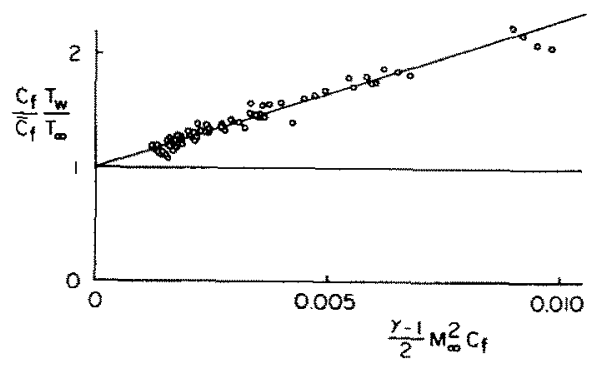

Frg. 3. Data of Fig. 2 in the coordinates of the substructure formulation. 
carefully derived from first principles. Among the immediate consequences of this transformation is the desired condition, in the form of an invariant called the law of corresponding stations. In essence, this law states that the product $C_{\mathrm{f}} \mathrm{Re}_{\theta}$ is the same at corresponding points in any two boundary-layer flows related by the transformation, provided only that the friction in both cases is Newtonian at the wall. No other important restrictions have to be put on the fluid properties or on the nature of the flows in question.

Once Fig. 1 has been established on relatively solid ground, the transformation next suggests a minor modification in the abscissa and a major modification in the ordinate of the figure. The change in abscissa is one that has been proposed independently by Matting et al. ${ }^{1}$ as a means of reconciling their measurements in air and helium; it is from $M_{\infty}$ to $(\gamma-1) M_{\infty}^{2} / 2$ (or, for practical purposes, from $M_{\infty}$ to $T_{w} / T_{\infty}$ or to $\left.q_{\infty} / p\right)$. This change not only removes the effect of variations in $\gamma$ for fixed Prandtl number, but also makes use of the fact that the curve of $T_{\infty} / T_{\mathrm{w}}$ against $(\gamma-1) M_{\infty}^{2} / 2$ is almost the only analytical curve that can be plotted in Fig. 1 without a detailed knowledge of the boundary-layer flow. Finally, the change in ordinate is a normalization of $C_{f} / \vec{C}_{f}$ with respect to this same thermodynamic parameter $T_{\infty} / T_{\mathrm{w}}$. These two changes of coordinate taken together lead to Fig. 2, in which there is still a noticeable scatter due to variations in Reynolds number from one experiment to another.

At this stage the inferences that can be drawn solely from the transformation and from measurements of gross properties of the boundary layer, such as the local surface friction, are exhausted. More information is needed, and it is assumed in Sec. IV that this information can be derived from the similarity law known as the law of the wall. The physical interpretation given to this law is that the sublayer flow in a turbulent boundary layer is characterized by a constant Reynolds number. An argument based on the transformation and on the data of Fig. 2 then suggests that this Reynolds number might be unaffected by compressibility if the density and viscosity are evaluated at a certain mean temperature defined by the transformation. Given this assumption, called here the sublayer hypothesis, it is found that the ordinate in Fig. 2 does not depend explicitly on conditions in the free stream, but only on conditions at or very near the

I F. W. Matting, D. Chapman, J. Nyholm, and A. Thomas, NASA Technical Report $\mathrm{R}-82$ (1960). wall. In order for the abscissa to have the same property, it suffices to change the independent variable from $(\gamma-1) M_{\infty}^{2} / 2$ to $(\gamma-1) M_{\infty}^{2} C_{f} / 2$ (or, for practical purposes, from $q_{\infty} / p$ to $\tau_{\mathrm{w}} / p$ ). The final correlation, as shown in Fig. 3, very nearly accounts for the dependence of the experimental data in Fig. 2 on Reynolds number.

For the sake of brevity the introductory material of this section is highly abridged, and several essential arguments have been oversimplified or omitted altogether. For example, it has been implied that the experimental data in Fig. 3 define a straight line. Strictly speaking, this statement is accurate only if the fluid is a perfect gas, if the viscosity is proportional to temperature, and if the stagnation temperature of the flow is constant. The analysis in the text, however, is not always limited to this special case, or even to the case of flow at constant pressure. On the contrary, the central element of the analysis at every point is a transformation that is remarkably free of restrictive conditions. Many of the relationships of this paper, therefore, may eventually be applicable to problems that have been less thoroughly studied experimentally than the classical problem of adiabatic flow at constant pressure past a smooth solid surface.

\section{Statement of the Problem}

The first objective is to find conditions under which the boundary-layer equations for compressible flow can be reduced to incompressible form by a suitable transformation. The second objective is to apply the transformation in question to the turbulent boundary layer. Neither problem is new, and almost all of the results to be derived for laminar flow have been obtained previously by Stewartson ${ }^{2}$ and others. More recently, several writers have attempted to extend the concept of a transformation to turbulent flow as well. However, little attention has been paid so far to several important issues raised by the latter application. Among these issues are the question of the complete identification of corresponding points and the question of the use of dimensionless similarity laws to describe the profiles of mean temperature and mean velocity. To avoid compromising these and other issues in advance, the transformation will be developed from first principles by means of arguments that are as rigorous and as general as possible.

The first problem stated in brief is this: The boundary-layer equations of mean motion for a compressible fluid of variable density $\rho(x, y)$ are given

\footnotetext{
${ }^{2}$ K. Stewartson, Proc. Roy. Soc. (London) A200, 84 (1949).
} 
in the form

$$
\begin{gathered}
\frac{\partial \rho u}{\partial x}+\frac{\partial \rho v}{\partial y}=0 \\
\rho u \frac{\partial u}{\partial x}+\rho v \frac{\partial u}{\partial y}=-\frac{d p}{d x}+\frac{\partial \tau}{\partial y}
\end{gathered}
$$

It is desired to find a formal transformation from $(x, y, u, v, p, \rho, \tau)$ to $(\bar{x}, \bar{y}, \bar{u}, \bar{v}, \bar{p}, \bar{\rho}, \bar{\tau})$ such that the original Eqs. (1.1)-(1.2) are reduced to the form

$$
\begin{gathered}
\frac{\partial \bar{u}}{\partial \bar{x}}+\frac{\partial \bar{v}}{\partial \bar{y}}=0, \\
\bar{\rho} \bar{u} \frac{\partial \bar{u}}{\partial \bar{x}}+\bar{\rho} \bar{v} \frac{\partial \bar{u}}{\partial \bar{y}}=-\frac{d \bar{p}}{d \bar{x}}+\frac{\partial \bar{\tau}}{d \bar{y}},
\end{gathered}
$$

with the parameter $\bar{\rho}$ independent of position in the barred flow.

The validity of these equations is not examined here. ${ }^{3}$ They evidently incorporate the usual boundary-layer approximation, in that the stresses in the fluid have been taken to be adequately represented by the static pressure $p$ (or $\bar{p}$ ) and by a single additional scalar component $\tau$ (or $\bar{\tau}$ ). However, the relationship of the latter quantity to the other variables of the problem has deliberately been left unspecified. Furthermore, no state or energy equation is included for the compressible flow. This formulation of the problem, while incomplete, has the advantage of being relatively free from assumptions that are either controversial or unduly restrictive. The present partial formulation of the problem also recognizes that the transformations for laminar and turbulent flow can be expected to have certain properties in common. These properties, to the extent that they can be inferred from Eqs. (1.1) through (1.4) without invoking the concepts of turbulent shearing stress and turbulent heat transfer, are therefore likely to have a relatively permanent significance.

\section{Physical Considerations}

In the text that follows frequent use is made of a physical principle whose importance does not seem to have been fully recognized in previous work on the transformation. The need for such a principle and the way in which it is to be applied are indicated in the following paragraphs.

${ }^{3}$ There is always the possibility that the usual boundarylayer approximation for the stress tensor may fail under certain conditions. In addition, there is room for argument about the importance in high-speed turbulent flows of various correlation terms (involving fluctuations in density, temperature, or viscosity) which are neglected here in order to treat the mean value of a product such as $\rho u$ or $\rho T$ (but not necessarily $\rho v$ ) as a product of mean values.
Suppose that a general transformation between Eqs. (1.1)-(1.2) and (1.3)-(1.4) has been established. Suppose further that the original compressible flow is characterized in some region by essentially constant density. This might be the case, for example, for adiabatic flow near the stagnation point of a blunt body, or in the upstrean part of a nozzle, or far downstream in a submerged jet. In such a. region the compressible equations can be reduced directly (i.e., without reference to the transformation) to incompressible form by the usual limiting process of retaining only the leading terms in expansions of appropriate dimensionless variables in powers of $M^{2}$. The resulting equations are identical in form with Eqs. (1.3)-(1.4), provided the density in the latter is evaluated at the appropriate stagnation condition in the original flow. A question then arises: Under these conditions, is it necessary for the transformation itself to reduce to the identity transformation?

In most previous work on the problem it has been tacitly assumed-e.g., by taking the reservoir conditions for the two flows to be the same - that the answer to this question is "yes." However, it then becomes necessary to suppose that the same fluid can behave entirely differently in the two flowslike a perfect gas, say, in one case, but like an incompressible liquid in the other. Whatever else may be said about this situation, it is unlikely that both flows can be observed experimentally. At the same time, it is certain that an experimental description of both flows is an essential part of any useful comparison in the case of turbulent flow.

The easiest way out of this dilemma is to avoid the presumption that the transformation should reduce to the identity transformation in a region where both flows are incompressible-or anywhere else, for that matter. On the contrary, it is more reasonable to suppose that the transformation should reduce instead to an affine transformation of the kind commonly used to investigate the question of similarity. ${ }^{4}$ This being so, it must be possible to choose the density $\bar{\rho}$ and the viscosity $\bar{\mu}$ for the barred flow quite arbitrarily. To this end the proposition will be taken as fundamental that the two flows represented by Eqs. (1.1)-(1.2) and (1.3)(1.4) must be treated in every respect as real physical

4 The author is aware that there exists no general affine transformation which is compatible with the experimental evidence and which allows the reduction of the turbulent boundary-layer equations to ordinary differential equations, as for laminar flow. The term similarity is used here in a broader sense not requiring uniform validity for the whole of the flow. 
flows, capable of being observed experimentally. It is a natural inference that corresponding variables should have the same physical dimensions and that the external and boundary conditions should be at least qualitatively the same in both problems. This principle has several useful consequences; for example, it allows the introduction of the parameter $\bar{\rho}$ on purely dimensional grounds.

In private discussions of this paper, the point has sometimes been raised that an application of the transformation to turbulent flow may involve unwarranted assumptions about the effect of compressibility on the mechanism of turbulence. That this need not be the case can be seen from an argument based in part on the physical principle just presented, but also in part on the fact that only one component of the stress tensor in addition to the pressure is present in the equations of motion considered here.

In any boundary-layer flow described by either of the momentum equations (1.2) or (1.4), the accelerations and the pressure forces are well-defined quantities that can be evaluated experimentally. The unbalance between these quantities may simply be taken to define an apparent shearing stress, and numerical values for the latter may thus be inferred from measurements of velocity and pressure. It is clearly irrelevant, for example, that this apparent stress may have a turbulent part that can be independently defined in terms of certain velocity fluctuations capable of being measured directly. If a transformation can be found to treat the acceleration and pressure terms in the equations, and if this transformation is required to be physically realistic, then it must follow without reference to any special definition of the shearing stresses that these stresses can also be treated by the transformation. This argument may seem to be academic, but it is probably the only one that can be relied on to provide an explicit transformation for the undefined shearingstress terms in Eqs. (1.1) through (1.4), and it is used for this purpose in Sec. II.

\section{THE FORMAL TRANSFORMATION}

\section{The Continuity Equation}

The development of the transformation begins by considering two stream functions $\psi$ and $\psi$, each of which is interpreted physically as an integral of mass rate rather than volume rate of flow. The two continuity Eqs. (1.1) and (1.3) are satisfied by introducing these two stream functions in the usual way:

$$
\rho u=\partial \psi / \partial y, \quad \rho v=-\partial \psi / \partial x,
$$

$$
\bar{\rho} \bar{u}=\partial \bar{\psi} / \partial \bar{y}, \quad \bar{\rho} \bar{v}=-\partial \bar{\psi} / \partial \bar{x},
$$

where the physical principle discussea in Sec. I requires an unspecified constant density $\bar{\rho}$ to be included in the second pair of equations in order to provide the same physical dimensions for $\bar{\psi}$ as for $\psi$.

The two stream functions are evidently constant on streamlines of their respective flows. In previous work on the transformation it has usually been assumed that these functions are the same at corresponding points and hence that streamlines in one flow are transformed into streamlines in the other. For reasons that will become apparent [see the remarks following Eq. (3.17)], it is desirable to avoid this assumption. The relationship between $\psi$ and $\bar{\psi}$ is therefore left open by writing

$$
\bar{\psi}(\bar{x}, \bar{y})=\sigma(x, y) \psi(x, y),
$$

where $\sigma$ is a completely unspecified function of $x$ and $y$.

\section{The Transport Terms}

The formal rule for the transformation of a derivative from coordinates $(\bar{x}, \bar{y})$ to coordinates $(x, y)$ may now be applied to the stream function $\Psi$, and the result expressed in terms of velocities with the aid of Eqs. (2.1) and (2.2). It is found that at corresponding points in the two flows,

$$
\begin{aligned}
& \rho u=\frac{1}{\sigma}\left(\bar{\rho} \bar{u} \frac{\partial \bar{y}}{\partial y}-\bar{\rho} \bar{v} \frac{\partial \bar{x}}{\partial y}\right)-\frac{\psi}{\sigma} \frac{\partial \sigma}{\partial y}, \\
& \rho v=\frac{1}{\sigma}\left(\bar{\rho} \bar{v} \frac{\partial \bar{x}}{\partial x}-\bar{\rho} \bar{u} \frac{\partial \bar{y}}{\partial x}\right)+\frac{\psi}{\sigma} \frac{\partial \sigma}{\partial x} .
\end{aligned}
$$

Further differentiation and substitution in the transport terms on the left-hand side of Eq. (1.2) yield, after some extremely tedious algebra, an identity that is given here in full for the record:

$$
\begin{aligned}
& \rho\left(u \frac{\partial u}{\partial x}+v \frac{\partial u}{\partial y}\right)=\left(\bar{u} \frac{\partial \bar{u}}{\partial \bar{x}}+\bar{v} \frac{\partial \bar{u}}{\partial \bar{y}}\right) \frac{\bar{\rho}^{2}}{\sigma^{2}} J(\bar{x}, \bar{y}) \frac{1}{\rho} \frac{\partial \bar{y}}{\partial y} \\
& -\left(\bar{u} \frac{\partial \bar{v}}{\partial \bar{x}}+\bar{v} \frac{\partial \bar{v}}{\partial \bar{y}}\right) \frac{\bar{\rho}^{2}}{\sigma^{2}} J(\bar{x}, \bar{y}) \frac{1}{\rho} \frac{\partial \bar{x}}{\partial y} \\
& +\bar{u}^{2} \frac{\bar{\rho}^{2}}{\sigma} J\left(\frac{1}{\sigma \rho} \frac{\partial \bar{y}}{\partial y}, \bar{y}\right) \\
& -\bar{u} \bar{v} \frac{\bar{\rho}^{2}}{\sigma}\left[J\left(\frac{1}{\sigma \rho} \frac{\partial \bar{x}}{\partial y}, \bar{y}\right)+J\left(\frac{1}{\sigma \rho} \frac{\partial \bar{y}}{\partial y}, \bar{x}\right)\right] \\
& +\bar{v}^{2} \frac{\bar{\rho}^{2}}{\sigma} J\left(\frac{1}{\sigma \rho} \frac{\partial \bar{x}}{\partial y}, \bar{x}\right)+
\end{aligned}
$$




$$
\begin{aligned}
& +\bar{\psi} \frac{\partial \bar{u}}{\partial \bar{y}} \frac{\bar{\rho}}{\sigma^{3}} J(\sigma, \bar{y}) \frac{1}{\rho} \frac{\partial \bar{y}}{\partial y}-\Psi \frac{\partial \bar{v}}{\partial \bar{x}} \frac{\bar{\rho}}{\sigma^{3}} J(\sigma, \bar{x}) \frac{1}{\rho} \frac{\partial \bar{x}}{\partial y} \\
& -\Psi \frac{\partial \bar{v}}{\partial \bar{y}} \frac{\bar{\rho}}{\sigma^{3}} J(\sigma, \bar{y}) \frac{1}{\rho} \frac{\partial \bar{x}}{\partial y}+\Psi \frac{\partial \bar{u}}{\partial \bar{x}} \frac{\bar{\rho}}{\sigma^{3}} J(\sigma, \bar{x}) \frac{1}{\rho} \frac{\partial \bar{y}}{\partial y} \\
& -\Psi \bar{u} \frac{\bar{\rho}}{\sigma^{3}}\left[J\left(\frac{1}{\rho} \frac{\partial \bar{y}}{\partial y}, \sigma\right)+\sigma J\left(\frac{1}{\sigma \rho} \frac{\partial \sigma}{\partial y}, \bar{y}\right)\right] \\
& +\bar{\psi} \bar{v} \frac{\bar{\rho}}{\sigma^{3}}\left[J\left(\frac{1}{\rho} \frac{\partial \bar{x}}{\partial y}, \sigma\right)+\sigma J\left(\frac{1}{\sigma \rho} \frac{\partial \sigma}{\partial y}, \bar{x}\right)\right] \\
& +\bar{\psi}^{2} \frac{1}{\sigma^{2}} J\left(\frac{1}{\sigma^{2} \rho} \frac{\partial \sigma}{\partial y}, \sigma\right) \text {. }
\end{aligned}
$$

In this identity the factors $J(A, B)$ denote the appropriate Jacobian $\partial(A, B) / \partial(x, y)$. In vector notation, $\mathbf{k} J(A, B)=\operatorname{grad} A \times \operatorname{grad} B$, with $\mathbf{k}$ the unit vector normal to the $x y$ plane. Hence $J(A, B)$ vanishes if $A$ or $B$ is a constant or, more generally, if $A$ is a function of $B$ alone.

The terms on the right-hand side of Eq. (2.6) have been arranged in descending order with respect to derivatives of $\bar{\psi}$. At least the first term must appear in the transformed momentum equation (1.4). The other terms are for the most part expendable, and the next step is to reduce Eq. (2.6) to manageable proportions by suitably restricting the dependence of the functions $\sigma, \bar{x}$, and $\bar{y}$ on $x$ and $y$.

For this purpose it is instructive to look at the behavior of the various terms in Eq. (2.6) outside a shear layer in the barred coordinates. Suppose first that there is a pressure gradient in the original flow, so that the left-hand side of Eq. (2.6) is nonzero but bounded for large values of $y$. If the regions outside the two shear flows are to correspond both physically and formally, there should also be a pressure gradient in the barred flow, and the righthand side of Eq. (2.6) should be nonzero but bounded for large values of $\bar{y}$. Now as far as the factors $\bar{\psi}$, $\bar{u}, \partial \bar{u} / \partial \bar{y}$, etc., are concerned, it appears that the term in $\bar{\psi}(\partial \bar{u} / \partial \bar{y})$ ordinarily vanishes for large values of $\bar{y}$ and that the terms in $D \bar{u} / D \bar{t}$ and $\bar{u}^{2}$ become at most functions of $\bar{x}$ only, whereas the remaining terms behave either like $\bar{y}$ or like $\bar{y}^{2}$. The simplest condition that ensures the proper behavior of the right-hand side of Eq. (2.6) is the requirement that each of the terms behaving like $\bar{y}$ or like $\bar{y}^{2}$ should vanish identically. This condition, in turn, is easily shown to be equivalent ${ }^{5}$ to requiring each of the quantities $\sigma, \bar{x}$, and $\partial \bar{y} / \rho \partial y$ to be independent of $y$.

${ }^{5}$ An identical result is obtained if the flow at infinity is assumed to be a uniform stream, but the condition on $\bar{y}$ seems to be unnecessary if the flow is bounded at infinity by a fluid at rest. A closer examination of this case, i.e., of the jet entering a stagnant fluid, is not undertaken here. Even

\section{The Momentum Equation}

At this point the transformation, except possibly for the jet, can be represented in the form

$$
\begin{aligned}
\bar{\psi} / \psi & =\sigma(x), \\
d \bar{x} / d x & =\xi(x), \\
(\bar{\rho} / \rho)(\partial \bar{y} / \partial y) & =\eta(x),
\end{aligned}
$$

where $\xi$ and $\eta$, like $\sigma$, are dimensionless functions of $x$ yet to be determined. Note that a second constant reference density is needed in Eq. (2.9) to give the proper physical dimension to the transformed variable $\bar{y}$, but that this parameter can be identified with $\bar{\rho}$ in Eq. (2.2) and any difference absorbed in $\eta(x)$.

The dependent variables $\bar{u}$ and $\bar{v}$ as derived from Eqs. (2.4) and (2.5) are now

$$
\begin{aligned}
& \bar{u}=(\sigma / \eta) u, \\
& \bar{v}=\frac{\rho \sigma}{\bar{\rho} \xi} v+\frac{\sigma}{\xi \eta} u \frac{\partial \bar{y}}{\partial x}-\frac{\psi}{\bar{\rho} \xi} \frac{d \sigma}{d x},
\end{aligned}
$$

and Eq. (2.6) can be written

$$
\begin{gathered}
\rho\left(u \frac{\partial u}{\partial x}+v \frac{\partial u}{\partial y}\right)=\bar{\rho}\left(\bar{u} \frac{\partial \bar{u}}{\partial \bar{x}}+\bar{v} \frac{\partial \bar{u}}{\partial \bar{y}}\right) \frac{\rho}{\bar{\rho}} \frac{\xi \eta^{2}}{\sigma^{2}} \\
+\frac{\rho u^{2}}{\eta / \sigma} \frac{d \eta / \sigma}{d x}+\frac{\psi}{\sigma} \frac{\partial u}{\partial y} \frac{d \sigma}{d x}=-\frac{d p}{d x}+\frac{\partial \tau}{\partial y}
\end{gathered}
$$

Of the three terms in Eq. (2.12) generated by the transformation of the transport terms in the original momentum equation (1.2) subject to the conditions (2.7) through (2.9), the first provides the transport terms in the transformed momentum equation (1.4). The other two terms, those in $\rho u^{2}$ and $\psi(\partial u / \partial y)$, therefore have to be suitably combined with the terms $d p / d x$ and $\partial \tau / \partial y$.

Consider now a companion equation to Eq. (2.12), obtained from the latter by solving for the transport

for the other cases mentioned the transformation in question may not be the most general or the most useful one which can be found; first, because the sufficient conditions used to bound the right-hand side of Eq. (2.6) for large $\bar{y}$ have not been shown to be necessary, and second, because these conditions have been applied not only for large $\bar{y}$ but throughout the barred flow. Furthermore, it has not been proved that the two flows involved in the transformation must always have the same character, i.e., that if one flow is a wake, so is the other; if one flow is a boundary layer with pressure gradient and mass transfer, so is the other; and so on. On the other hand, the transformation considered here is more general than any that has been considered previously, and has several properties that will prove to be useful in any study of turbulent flow. The possibility of a more general transformation is therefore not investigated further. 
terms in barred coordinates and using Eq. (1.4):

$$
\begin{aligned}
& \bar{\rho}\left(\bar{u} \frac{\partial \bar{u}}{\partial \bar{x}}+\bar{v} \frac{\partial \bar{u}}{\partial \bar{y}}\right) \\
& =\frac{\bar{\rho}}{\rho} \frac{\sigma^{2}}{\xi \eta^{2}}\left(-\frac{d p}{d x}+\frac{\partial \tau}{\partial y}-\frac{\rho u^{2}}{\eta / \sigma} \frac{d \eta / \sigma}{d x}-\frac{\psi}{\sigma} \frac{\partial u}{\partial y} \frac{d \sigma}{d x}\right) \\
& =-\frac{d \bar{p}}{d \bar{x}}+\frac{\partial \bar{\tau}}{\partial \bar{y}} .
\end{aligned}
$$

The second equality in Eq. (2.13) is a relationship involving primarily pressures and shearing stresses. The right-hand side of this equality consists of two terms, $d \bar{p} / d \bar{x}$ and $\partial \bar{\tau} / \partial \bar{y}$. According to the physical principle underlying the present transformation, the first of these terms must depend only on $\bar{x}$ (or $x$ ), and the second must vanish for large $\bar{y}$ (or $y$ ). It is therefore necessary to write the left-hand side in the same fashion, and it follows immediately that the transformed quantities $\bar{p}$ and $\bar{\tau}$ have to be defined by

$$
\frac{d \bar{p}}{d \bar{x}}=\frac{\bar{\rho} \sigma^{2}}{\xi \eta^{2}}\left(\frac{1}{\rho_{\infty}} \frac{d p}{d x}+\frac{u_{\infty}^{2}}{\eta / \sigma} \frac{d \eta / \sigma}{d x}\right)
$$

and by

$$
\begin{gathered}
\frac{\partial \bar{\tau}}{\partial \bar{y}}=\frac{\bar{\rho} \sigma^{2}}{\xi \eta^{2}}\left[\frac { 1 } { \rho } \left(\frac{\partial \tau}{\partial y}\right.\right. \\
\left.-\frac{\psi}{\sigma} \frac{\partial u}{\partial y} \frac{d \sigma}{d x}\right)+\frac{d p}{d x}\left(\frac{1}{\rho_{\infty}}-\frac{1}{\rho}\right) \\
\left.+\frac{1}{\eta / \sigma} \frac{d \eta / \sigma}{d x}\left(u_{\infty}^{2}-u^{2}\right)\right],
\end{gathered}
$$

respectively.

The slightly awkward form of the last two relationships is justified by their generality. Neither an equation of state nor an energy equation for the compressible flow has yet been cited. Furthermore, it has not been necessary to define the shearing stresses $\tau$ and $\bar{\tau}$ explicitly, and no conditions have been imposed that require the presence of a solid boundary. The fact that the form of the transformation can be established under such general conditions is due largely to the emphasis that is put here on physical considerations. In particular, the argument relating to Eqs. (2.12) through (2.15) can be recognized as a repetition of the argument outlined at the end of Section I in connection with the irrelevvance of the shearing-stress mechanism.

However, the transformation is not in a useful form at this juncture, inasmuch as nothing is known about the properties of the three scaling functions $\sigma(x), \xi(x)$, and $\eta(x)$, which are so far defined only by Eqs. (2.7) through (2.9). In Sec. III it is shown that some information about these functions can be obtained at a quite reasonable cost, measured in terms of generality. First, however, one important and useful property of the transformation is demonstrated in the following paragraphs for the special case of a flow bounded by a smooth wall.

\section{The Law of Corresponding Stations}

So far there has been neither need nor opportunity to introduce a viscosity $\bar{\mu}$ for the barred flow. An obvious and natural means for introducing this parameter is the use of the condition $\bar{\tau}=\mu(\partial \vec{u} / \partial \vec{y})$ for Newtonian friction, provided, of course, that this condition is appropriate. In the case of a free turbulent flow such as a jet or wake, or in the case of a boundary layer on a rough wall, the condition is evidently not appropriate. The viscosity is in a certain sense an artificial parameter, and the Reynolds number plays at most a secondary role in the description of the mean motion. When these cases are excluded, there remains the case of a boundary layer on a smooth wall, and here the viscosity clearly plays a primary role.

Consider such a boundary-layer flow, where the wall is assumed for convenience to be at $y=\bar{y}=0$. Whether the flow is laminar or turbulent, and whether or not there is mass transfer through the surface, the conditions $\bar{\tau}_{\mathrm{w}}=\bar{\mu}(\partial \bar{u} / \partial \bar{y})_{\mathrm{w}}$ and $\tau_{\mathrm{w}}=$ $(\mu \partial u / \partial y)_{w}$ can be expected to hold at the wall. The transformation Eqs. (2.9) and (2.10) for $u$ and $y$ then require $\bar{\tau}_{\mathrm{w}}$ and $\tau_{\mathrm{w}}$ to be related by

$$
\bar{\tau}_{\mathrm{w}}=\frac{\bar{\rho} \bar{\mu}}{\rho_{\mathrm{w}} \mu_{\mathrm{w}}} \frac{\sigma}{\eta^{2}} r_{\mathrm{w}} .
$$

Local friction coefficients $C_{\mathrm{f}}$ and $\bar{C}_{\mathrm{f}}$, defined in the usual way, are then found to satisfy

$C_{\mathrm{f}}=\frac{2 \tau_{\mathrm{w}}}{\rho_{\infty} u_{\infty}^{2}}=2 \frac{\sigma \mu_{\mathrm{w}}}{\bar{\mu}} \frac{\rho_{\mathrm{w}}}{\rho_{\infty}} \frac{\bar{\tau}_{\mathrm{w}}}{\bar{\rho} \bar{u}_{\infty}^{2}}=\frac{\sigma \mu_{\mathrm{w}}}{\bar{\mu}} \frac{\rho_{\mathrm{w}}}{\rho_{\infty}} \bar{C}_{\mathrm{f}}$,

where the subscript $\infty$ refers to the external flow. Upon introducing the conventional momentum thickness

$$
\theta=\int_{0}^{\infty} \frac{\rho u}{\rho_{\infty} u_{\infty}}\left(1-\frac{u}{u_{\infty}}\right) d y
$$

and the corresponding thickness $\bar{\theta}$, it appears that

$$
\bar{\theta}=\int_{0}^{\infty} \frac{\bar{u}}{\bar{u}_{\infty}}\left(1-\frac{\bar{u}}{\bar{u}_{\infty}}\right) d \bar{y}=\frac{\rho_{\infty}}{\bar{\rho}} \eta \theta .
$$

Finally, Reynolds numbers based on $\theta$ and $\bar{\theta}$ may be defined in the usual way and connected by

$$
\operatorname{Re}_{\theta}=\frac{\rho_{\infty} u_{\infty} \theta}{\mu_{\infty}}=\frac{\bar{\mu}}{\sigma \mu_{\infty}} \frac{\bar{\rho} \bar{u}_{\infty} \bar{\theta}}{\bar{\mu}}=\frac{\bar{\mu}}{\sigma \mu_{\infty}} \overline{\operatorname{Re}}_{\bar{\theta}}
$$


where $\bar{\mu}$ in Eq. (2.20) is again to be interpreted as the viscosity of the barred fluid. When $\sigma$ is eliminated between Eqs. (2.17) and (2.20), it is seen that

$$
\bar{C}_{\mathrm{f}} \overline{\operatorname{Re}}_{\bar{\theta}}=\frac{\rho_{\infty} \mu_{\infty}}{\rho_{\mathrm{w}} \mu_{\mathrm{w}}} C_{\mathrm{f}} \operatorname{Re}_{\theta},
$$

and this is the desired result. The relationship (2.21), which seems to have been overlooked in previous work on the transformation, is called the law of corresponding stations for the boundary layer on a smooth wall. A special case of this law is well known in the theory of similar solutions for the laminar boundary layer. However, it should be emphasized that Eq. (2.21) has been derived here by considering the structure of the incomplete equations of motion rather than the behavior of certain special solutions of these equations. This law is obviously of considerable value in any use of experimental data to test the validity of the present transformation for the case of turbulent flow.

\section{THE THREE SCALING FUNCTIONS $\boldsymbol{d}, \xi$, AND n}

\section{Laminar Flow}

The remaining step in completing the transformation is the specification of the three scaling functions $\sigma, \xi$, and $\eta$, which determine the transformation of the stream function $\psi$ and the coordinates $x$ and $y$, respectively. These functions are studied first for the laminar case, with the object of providing at least a qualitative model for the later discussion of turbulent flow.

If the flow is laminar, the shearing stresses $\tau$ and $\bar{\tau}$ are no longer unspecified, being given by $\mu(\partial u / \partial y)$ and by $\bar{\mu}(\partial \bar{u} / \partial \bar{y})$ throughout the two flows related by the transformation. It follows that Eq. (2.16) is valid without the subscript $w$,

$$
\bar{\tau}=\frac{\bar{\rho} \bar{\mu}}{\rho \mu} \frac{\sigma}{\eta^{2}} \tau,
$$

and therefore that

$$
\frac{\partial \bar{\tau}}{\partial \bar{y}}=\frac{\bar{\rho}^{2} \bar{\mu} \sigma}{\rho^{2} \mu \eta}\left(\frac{\partial \tau}{\partial y}-\frac{\tau}{\rho \mu} \frac{\partial \rho \mu}{\partial y}\right) .
$$

This indirect transformation (3.2) for $\partial \bar{\tau} / \partial \vec{y}$, which is a consequence of the separate transformations for $u$ and $y$, must be consistent with the direct and more general transformation (2.15), which does not assume Newtonian friction. The simplest way to ensure consistency, although perhaps not the only one, is to require the two expressions for $\partial \bar{\tau} / \partial \bar{y}$ to be identical. This is certainly the case if

$$
\begin{gathered}
d \sigma / d x=0 \\
\partial \rho \mu / \partial y=0
\end{gathered}
$$

and

$$
\frac{d p}{d x}\left(\frac{1}{\rho_{\infty}}-\frac{1}{\rho}\right)+\frac{1}{\eta / \sigma} \frac{d \eta / \sigma}{d x}\left(u_{\infty}^{2}-u^{2}\right)=0,
$$

in which case it also follows that

$$
\frac{\xi}{\sigma \eta}=\frac{\rho \mu}{\bar{\rho} \bar{\mu}}=\frac{\rho_{\mathrm{w}} \mu_{\mathrm{w}}}{\bar{\rho} \bar{\mu}}=\frac{\rho_{\infty} \mu_{\infty}}{\bar{\rho} \bar{\mu}}
$$

The condition given by Eq. (3.4) is evidently satisfied for a perfect gas having the usual equation of state

$$
p=\rho R T
$$

with $R=c_{p}-c_{\varepsilon}=$ constant, if, in addition, the viscosity is proportional to the temperature. The condition given by Eq. (3.5) is automatically satisfied if there is no pressure gradient in either flow, since Eqs. (2.14) and/or (2.10) then require the ratio $\eta / \sigma$ to be independent of $x$. More generally, however, the condition given by Eq. (3.5) can be rewritten after a separation of variables as

$$
\frac{2}{\eta / \sigma} \frac{d \eta / \sigma}{d p}=\frac{1 / \rho-1 / \rho_{\infty}}{\frac{1}{2} u_{\infty}^{2}-\frac{1}{2} u^{2}}=c(x) .
$$

If the compressible fluid is a perfect gas, the second equality immediately suggests that $c(x)$ should be taken as $R / c_{p} p(x)$. Equation (3.8) then yields the two conditions

$\frac{1}{2} u_{\infty}^{2}+c_{p} T_{\infty}=c_{p} T_{0 \infty}=\frac{1}{2} u^{2}+c_{p} T=c_{p} T_{0}=$ constant

and

$$
\eta^{2} / \sigma^{2}=T_{\infty} / \bar{T}
$$

where $\bar{T}$ in the second equation denotes an arbitrary constant of integration with the dimensions of temperature. Both of the last two expressions make indirect use of the condition $p p_{\infty}^{-\gamma}=$ constant for an isentropic external flow, with $\gamma=c_{p} / c_{v}=$ constant.

As might be expected. these results for laminar flow are simply a restatement of the well-known conditions (perfect gas, $\mu / T$ constant, $p$ or $T_{0}$ constant) under which the momentum and continuity equations can be uncoupled from the energy equation and integrated separately in barred co- 
ordinates. ${ }^{6}$ The problem of the laminar boundary layer was first approached from this direction by Dorodnitsyn. ${ }^{9,10}$ In Dorodnitsyn's formulation, however, the omission of the scaling function denoted by $\eta(x)$ led to the appearance of a factor $T / T_{\infty}$ multiplying the transformed pressure-gradient term, so that the reduction to incompressible form was achieved only for the case $\eta=$ constant or $d p / d x=0$. Cope and Hartree, ${ }^{11}$ in the course of some research in a different direction, independently proposed a partial transformation in the spirit of Eq. (2.9) for the normal coordinate $y$. Howarth, ${ }^{12}$ again independently, also introduced a partial transformation equivalent to Eq. (2.9), but with $\eta(x)$ proportional to $1 /[p(x)]^{\frac{1}{2}}$ rather than to $\left[T_{\infty}(x)\right]^{\frac{1}{2}}$. Howarth also elected to preserve the streamwise velocity component $u$ rather than the stream function $\psi$ at corresponding points, and the pressure-gradient term again emerged in quite awkward form in the final

${ }^{6}$ The transformation derived in this report has been designed specifically for turbulent flow, and strict attention has been paid from the beginning to the physical principle that it must be possible to observe experimentally any flow whose properties are supposed to be known. This principle, which makes it essential to keep in close touch with the physical variables and with the conservation laws connecting them, is most appropriate for problems that cannot be treated by analytical means. For problems that can be so treated, on the other hand, almost any transformation or change of variable is acceptable if it leads to equations that are mathematically more tractable than the equations of motion in their original form. The principle involved in such cases is a mathematical principle, however, not a physical one, and may be as much out of place in discussions of turbulence as is the physical principle in most discussions of laminar flow.

An example of the use (and misuse) of the alternative mathematical principle is supplied by the transformation first proposed by Stewartson in Ref. 2 and later cited for turbulent flow by some writers. The transformation in question may be obtained by simply dropping the subscript $\infty$ in Eqs. (2.14) and (2.15) of Sec. II. For a perfect gas the quantity in parentheses in Eq. (2.14) can then be written as $R T_{0} d p / p d x$, where $T_{0}$ is the local stagnation temperature. In this formulation the principle of physical existence for the barred flow seems to require $T_{0}=$ constant if $d p / d x \neq 0$ as before, whether the flow is laminar or turbulent. However, this requirement is entirely without force for the turbulent case, because the physical principle is being applied one stage too late in the argument. Even for laminar flow the requirement in question is largely irrelevant, and Stewartson's transformation has been used (e.g., by Cohen and Reshotko? and by $\mathrm{Coles}^{8}$ ) to obtain physically meaningful similarity solutions for laminar flow with heat transfer and pressure gradient, in spite of the fact that the transformed equations do not correspond to any flow that can be observed experimentally.

${ }^{7}$ C. B. Cohen and E. Reshotko, NACA Technical Note TN 3325 (1955).

8 D. Coles, in Proceedings of the Heat Transfer and Fluid Mechanics Institute (Stanford University Press, Stanford, California, 1957), p. 119.

${ }^{9}$ A. Dorodnitsyn, Compt. Rend. (Doklady) URSS 34, $213(1942)$.

${ }_{10}$ A. Dorodnitsyn, Prikl. Matem. Mekh. 6, 449 (1942).

11 W. F. Cope and D. Hartree, Phil. Trans. Roy. Soc. (London) A241, 1 (1948).

${ }_{12}$ L. Howarth, Proc. Roy. Soc. (London) A194, 16 (1948). equation for the transformed stream function. Stewartson (Ref. 2), in continuing Howarth's work, chose to preserve the stream function instead and to use a suitable scaling function $\eta(x)$, and thus arrived at a laminar transformation equivalent to the transformation considered here. Minor differences include Stewartson's use of boundary-layer variables $y /(\nu)^{\frac{1}{2}}$ and $v /(\nu)^{\frac{1}{2}}$ [but not $\left.\psi /(\nu)^{\frac{1}{2}}\right]$, and his early consolidation of the various scaling functions. The general conditions under which the compressible equations of motion can be reduced to incompressible form were also discovered simultaneously by Illingworth, ${ }^{13}$ working in von Mises' coordinates $x, \psi$ rather than in physical coordinates $x, y$.

\section{Turbulent Flow at Constant Pressure}

If the flow is turbulent, the arguments leading to Eqs. (3.3), (3.6), and (3.10) have to be discarded and a fresh beginning made. For the present the discussion is limited to the case of flow at constant pressure, for which the general transformation (2.15) for $\partial \tau / \partial y$ can be integrated in the relatively simple form

$$
\frac{\xi \eta}{\sigma^{2}} \bar{\tau}=\tau+\frac{1}{\sigma} \frac{d \sigma}{d x} \int_{y}^{\infty} \psi \frac{\partial u}{\partial y} d y .
$$

At the wall this expression becomes

$$
\frac{\xi \eta}{\sigma^{2}} \bar{\tau}_{\mathrm{w}}=\tau_{\mathrm{w}}+\frac{1}{\sigma} \frac{d \sigma}{d x}\left(\rho_{\infty} u_{\infty}^{2} \theta+\psi_{\mathrm{w}} u_{\infty}\right)
$$

where the notation makes use of an identity,

$$
\int_{0}^{\infty} \psi \frac{\partial u}{\partial y} d y=\rho_{\infty} u_{\infty}^{2} \theta+\psi_{\mathrm{w}} u_{\infty}
$$

which can be obtained from the definition (2.18) for $\theta$ by putting $\partial \psi / \partial y$ for $\rho u$ and integrating by parts. These relationships are independent of the state and energy equations and the viscosity law, and they can be applied to rough as well as to smooth surfaces as long as $\tau_{\mathrm{w}}$ and $\bar{\tau}_{\mathrm{w}}$ are interpreted as tangential force per unit area.

For the particular case of a smooth wall, the shearing stresses at $y=0$ must also be connected by Eq. (2.16),

$$
\frac{\rho_{\mathrm{w}} \mu_{\mathrm{w}}}{\bar{\rho} \bar{\mu}} \frac{\eta^{2}}{\sigma} \bar{\tau}_{\mathrm{w}}=\tau_{\mathrm{w}}
$$

Moreover, if there is no mass transfer, the combination $\psi_{\mathrm{w}}(d \sigma / d x)$ in $\mathrm{Eq}$. (3.12) may be taken as

${ }^{13}$ C. R. Illingworth, Proc. Roy. Soc. (London) A199, $533(1949)$. 
zero [see Eq. (2.11) evaluated at $y=0$ ]. Equations (3.12) and (3.14), together with the momentumintegral equation $\tau_{\mathrm{w}}=\rho_{\infty} u_{\infty}^{2}(d \theta / d x)$, then imply

$$
\frac{\xi}{\sigma \eta} \frac{\bar{\rho} \bar{\mu}}{\rho_{\mathrm{w}} \mu_{\mathrm{w}}}-1=\frac{\rho_{\infty} u_{\infty}^{2} \theta}{\tau_{\mathrm{w}} \sigma} \frac{d \sigma}{d x}=\frac{\theta}{\sigma} \frac{d \sigma}{d \theta} .
$$

This expression provides virtually the last point of contact between the analyses for laminar and for turbulent flow. For laminar flow the two sides of Eq. (3.15) vanish identically, by virtue of the condition $d \sigma / d x=0$, or $\sigma=$ constant, which was originally imposed by requiring Newtonian friction away from the wall. For turbulent flow, on the other hand, the experimental evidence to be cited shortly implies that $d \sigma / d \theta$ is not zero, so that Eq. (3.15) is no longer a trivial identity but a genuine condition connecting the three scaling functions with each other and with the ostensible data of the problem. This single condition for turbulent flow, moreover, differs from the corresponding two conditions (3.3) and (3.6) for laminar flow in one important respect, since Eq. (3.15) involves not only quantities such as $\mu_{\mathrm{w}}$ and $u_{\infty}$, which are usually regarded as part of the given data for a particular problem, but also quantities such as $\tau_{\mathrm{w}}(x)$ and $\theta(x)$, which are usually regarded as part of the solution.

Finally, if $\sigma$ is in fact a function of $x$ for a turbulent boundary layer at constant pressure, it follows from an argument inverse to the one employed for laminar flow that $\tau$ is not transformed like $\mu(\partial u / \partial y)$ except at the wall. Consequently there seems to be no reason in the case of turbulent flow to require the compressible fluid to be a perfect gas or to require the viscosity to be proportional to temperature. Neither is there any reason to suppose that the energy equation plays any significant part in the direct transformation, at least when the pressure gradient is zero.

\section{The Function $\delta(x)$ for Turbulent Flow}

At this point it is convenient to consider briefly some experimental investigations that have recently been carried out in adiabatic turbulent boundary layers in supersonic flow at constant pressure. The discussion is limited to experiments that include measurements of local surface friction as well as measurements of local mean-velocity distribution. That the number of such experiments is by this time usefully large is evident from Fig. 4, in which are collected all of the local friction data so far obtained in air.

In what follows, any slight discrepancies in Fig. 4 between the data of various observers are overlooked

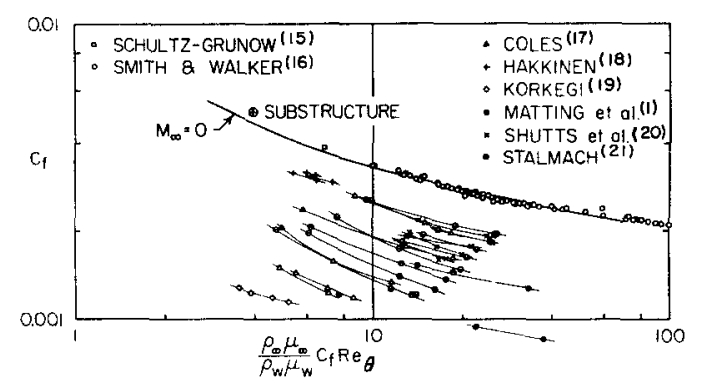

FIG. 4. Floating-element measurements of local surface friction in air. Data from Refs. 1, 15-21.

in order to assume that there is a unique relationship among the three parameters $C_{f}, \operatorname{Re}_{\theta}$, and $M_{\infty}$, at least for air at typical wind-tunnel temperatures. The main objective of this paper is to determine the relationship in question. The immediate problem, however, is to determine empirically the value of the combination $\bar{\mu} / \sigma \mu_{\mathrm{w}}$, which is expressed in terms of readily measured quantities by Eq. (2.17),

$$
\bar{\mu} / \sigma \mu_{\mathrm{w}}=\left(\rho_{\mathrm{w}} / \rho_{\infty}\right)\left(\bar{C}_{\mathrm{f}} / C_{\mathrm{f}}\right) .
$$

The local friction coefficients $C_{f}$ and $\bar{C}_{f}$ in Eq. (3.16) are supposedly connected by the law of corresponding stations,

$$
\bar{C}_{f} \overline{\operatorname{Re}}_{\bar{\theta}}=\frac{\rho_{\infty} \mu_{\infty}}{\rho_{\mathrm{w}} \mu_{\mathrm{w}}} C_{\mathrm{f}} \operatorname{Re}_{\theta}
$$

and the coordinates of Fig. 4 have been chosen accordingly. ${ }^{14}$ In order to use Eq. (3.17) to determine $\bar{C}_{\mathrm{f}}$ when $M_{\mathrm{o}}, C_{\mathrm{f}}, \mathrm{Re}_{\theta}$, and the fluid properties are known, it is necessary to know the dependence of $\bar{C}_{\mathrm{f}}$ on $\overline{\mathrm{Re}}_{\bar{\theta}}$ (or on $\bar{C}_{\mathrm{f}} \overline{\mathrm{Re}}_{\bar{\theta}}$ ) in incompressible flow, especially at the lower Reynolds numbers. This depend-

${ }_{14}$ Although Fig. 4 is equivalent to Fig. 1 of Sec. I, the earlier figure emphasizes the dependence of skin friction on Mach number, whereas Fig. 4 emphasizes the dependence on Reynolds number. In a different sense Fig. 5 is equivalent to Fig. 2, since the coordinates in one figure are essentially reciprocals of the coordinates in the other. In this case, however, the earlier treatment of the data is simply a normalization of $C_{t} / \bar{C}_{f}$ with respect to temperature ratio, whereas Fig. 5 represents an inquiry into the properties of the scaling function $\sigma(x)$ of the transformation.

In the construction of Figs. 4 and 5 , the ratio $T_{\mathrm{w}} / T_{\infty}$ has been referred to a recovery factor of 0.885 in all cases where no experimental value is given in the original reference. Values of viscosity have been taken from standard NBSNACA tables. The usual practice has been followed of assuming constant stagnation temperature $T_{0}$ for the purpose of computing $u(y)$ and $\rho(y)$ and thus $\theta(x)$ when only $M(y)$ can be inferred from the measurements. The floatingelement data have been corrected for gap effect by using the combined area of element and gap rather than the area of the element alone to relate force and shearing stress. Finally, the values of $\bar{C}_{f}$ for low-speed flow have been estimated by fitting measured mean-velocity profiles to the law of the wall. 


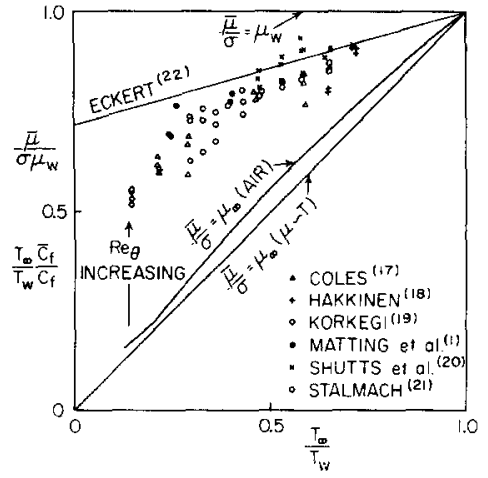

Frg. 5. Empirical evaluation of the parameter $\bar{\mu} / \sigma \mu_{\mathrm{W}}$ of the transformation. Data from Refs. 1, 17-22.

ence is also shown in Fig. $4 .^{1,15-21}$ Although the curve drawn to represent the incompressible data can be described analytically with the aid of certain similarity laws, this curve should be viewed for the present as a strictly empirical result, and the calculations involving Eqs. (3.16) and (3.17) should be viewed as nothing more complicated than a comparison of one set of experimental data with another.

When the parameter $\bar{\mu} / \sigma \mu_{\mathrm{w}}$ determined with the aid of Eq. (3.16) is plotted against, say, the temperature ratio $T_{\infty} / T_{\mathrm{w}}$, as shown in Fig. $5,{ }^{1,17-22}$ it is found that the data at a given Mach number show a weak but definite dependence on Reynolds number, quite apart from unavoidable experimental scatter. The necessary conclusion is that the combination $\bar{\mu} / \sigma \mu_{\mathrm{w}}$, which is here equivalent to the ratio $C_{\mathrm{f}} / \bar{C}_{\mathrm{f}}$, is not only a function of Mach number for these experiments, but also a function of Reynolds number. Because $\mu_{w}$ and $\bar{\mu}$ are constants for these data, it can be concluded that $\sigma$ must be treated as a function of Reynolds number and hence of $x$ for the flows in question.

\section{THE NOTION OF A SUBSTRUCTURE}

\section{Recapitulation}

Up to this point the search for a transformation capable of reducing the compressible turbulent boundary-layer equations to incompressible form

${ }^{15}$ F. Schultz-Grunow, Luftfahrtforsch. 17, 239 (1940).

${ }_{16}$ D. W. Smith and J. Walker, NACA Technical Note TN 4231 (1958).

$17 \mathrm{D}$. Coles, thesis, California Institute of Technology (1953).

${ }_{18} \mathrm{R}$. Hakkinen, thesis, California Institute of Technology (1954).

${ }^{19}$ R. H. Korkegi, thesis, California Institute of Technology (1954).

${ }_{20}$ W. H. Shutts, W. Hartwig, and J. Weiler, University o Texas Report DRL-364 (1955).

${ }_{21}$ C. J. Stalmach, Jr., University of Texas Report DRL-410 (1958).

${ }_{22}$ E. R. G. Eckert, Wright Air Development Center Technical Report 54-70 (1954). has been carried out in three steps. The physical basis of the transformation was laid down in Sec. I; the transformation itself was formally constructed in Sec. II; and a preliminary study was made in Sec. III of the three scaling functions whose properties must be known before the transformation can be applied to a given problem.

For turbulent flow, even for the special case of turbulent fiow at constant pressure, only two independent relationships have so far been found for the three scaling functions $\sigma, \xi$, and $\eta$. One of these relationships,

$$
\sigma / \eta=\text { const }=\bar{u}_{\infty} / u_{\infty},
$$

say, follows from the condition of constant pressure; the other,

$$
\frac{\xi}{\eta}=\frac{\rho_{\mathrm{w}} \mu_{\mathrm{w}}}{\bar{\rho} \bar{\mu}} \frac{d \sigma \theta}{d \theta},
$$

then follows from the additional condition of Newtonian friction at the wall. For flow in a wake, the condition that replaces Eq. (4.2) is evidently $\eta=$ constant $=\bar{\rho} \bar{\theta} / \rho_{\infty} \theta$, say, in view of Eq. (2.19). None of these conditions implies any restriction on the state or energy equations or on the viscosity law.

Before the transformation can be applied to turbulent flow, it is necessary to find a third condition corresponding to Eqs. (4.1) and (4.2). The form of this third condition can be expected to vary with the circumstances of the problem. For example, the condition $\sigma=$ constant, which has already been shown to be generally valid for laminar flow or for a turbulent wake, must be rejected on experimental grounds when the flow is an adiabatic turbulent boundary layer at constant pressure. For the latter case, according to Fig. 5 , the quantity $\sigma \mu_{w} / \bar{\mu}$ depends both on Mach number and on Reynolds number. The analytical nature of this dependence is so far unknown, and presumably has to be established with the aid of some physical argument beyond those already cited. Whatever the nature of this argument may be, it should be clear that the problem that now has to be solved is a different problem from the one originally formulated in Sec. I. The issue is no longer the transformation of the equations of mean motion to incompressible form. Instead, the issue is now the search for a hypothesis which allows this transformation to be applied to the turbulent boundary layer. Inasmuch as a great variety of physical considerations might be taken as characteristic of turbulent boundary-layer flow, an equally great variety of arguments might serve to complete the transforma- 
$\operatorname{tion}^{23}$ by determining the scaling function $\sigma(x)$. It goes without saying that different writers may prefer different arguments for this purpose, and therefore that the particular argument that follows can and should be judged by less critical standards than those used to judge the material of Secs. I through III.

\section{The Law of the Wall}

The physical considerations that are taken here as characteristic of turbulent flow near a smooth wall are contained in an empirical similarity relationship known as the law of the wall. For incompressible flow this law has the form

$$
\frac{\bar{u}}{\left(\bar{\tau}_{\mathrm{w}} / \bar{\rho}\right)^{\frac{1}{2}}}=f\left(\frac{\bar{\rho} \bar{y}\left(\bar{\tau}_{\mathrm{w}} / \bar{\rho}\right)^{\frac{1}{2}}}{\bar{\mu}}\right)=f(z) .
$$

When translated into compressible variables with the aid of the transformation formulas for $u$ and $y$, this statement becomes

$$
\left(\frac{\sigma \mu_{\mathrm{w}}}{\bar{\mu}}\right)^{\frac{1}{2}} \frac{u}{\left(\tau_{\mathrm{w}} / \rho_{\mathrm{w}}\right)^{\frac{1}{2}}}=f\left[\left(\frac{\sigma \mu_{\mathrm{w}}}{\bar{\mu}}\right)^{\frac{1}{2}} \frac{\left(\tau_{\mathrm{w}} / \rho_{\mathrm{w}}\right)^{\frac{1}{2}}}{\mu_{\mathrm{w}}} \int_{0}^{y} \rho d y\right] \text {. }
$$

If the flow is incompressible, two alternative physical interpretations are available for the relationship (4.3). One is that the edge of the viscous sublayer, defined by specifying a suitable numerical value for the function $f$ or its argument $z$, is a streamline of the mean flow. The other is that the sublayer Reynolds number, defined by specifying a suitable numerical value for the product $f z$, say, is a constant. Both of these interpretations are expressed in terms that allow their immediate formal extension to compressible flow. However, the first interpretation has now to be disqualified on the ground that it requires the scaling function $\sigma$ to be a constant. ${ }^{24}$

${ }^{23}$ It is important to note that the end to be served is the completion of the transformation, not the completion of the equations of motion through the introduction of an explicit relationship connecting the shearing stresses $\tau$ and $\bar{\tau}$ with the other dependent or independent variables, as in the case of laminar flow. On the contrary, the use of any such relationship is quite likely to prejudice the concepts of turbulent shearing stress and turbulent heat transfer in much the same way that these concepts are prejudiced by the mixing analogies of the older literature.

24 The author has previously attempted an extension of the first interpretation to compressible flow, ${ }^{25}$ using only part of the full transformation given here. This earlier paper, in which the quantity now called $\sigma \mu_{w} / \bar{\mu}$ was denoted by $\rho_{\tau} / \rho_{\mathrm{x}}$, includes a demonstration that this quantity (and hence $\sigma$ ) must be independent of $x$ if $u /\left(\tau_{w} / \rho_{\tau}\right)^{t}=f$ is constant on mean streamines of the compressible flow. Except for the one negative conclusion just quoted, the discussion of compressibility in Ref. 25 may therefore be suppressed.

${ }_{25}$ D. Coles, 50 Jahre Grenzschichtforschung, edited by H. Görtler and W. Tollmien (Vieweg, Braunschweig, Germany, 1955), p. 153.
The second interpretation, which appears to be free from this restriction, implies for compressible flow

$\operatorname{Re}_{s}=f_{s} z_{s}=\frac{\bar{\rho} \bar{u}_{s} \bar{y}_{s}}{\bar{\mu}}=\frac{\sigma u_{s}}{\bar{\mu}} \int_{0}^{y_{s}} \rho d y=$ constant. (4.5)

\section{The Sublayer Hypothesis}

The last equation may be manipulated in such a way as to suggest a possible connection between the ratio $\bar{\mu} / \sigma$ and the properties of the flow in the sublayer. First, define a mean density $\rho_{\mathrm{s}}$ for the sublayer as

$$
\rho_{s}=\frac{1}{y_{\mathrm{s}}} \int_{0}^{y_{\mathrm{s}}} \rho d y,
$$

whereupon Eq. (4.5) can be written

$$
\operatorname{Re}_{\mathrm{s}}=\rho_{\mathrm{a}} u_{\mathrm{s}} y_{\mathrm{s}} /(\bar{\mu} / \sigma)=\text { constant. }
$$

Further, suppose that an equation of state is given in the form $T=T(\rho, p)=T(\rho)$ at constant $p$, so that a definite mean temperature $T_{\mathrm{s}}=T\left(\rho_{\mathrm{s}}\right)$ can be associated with the mean density defined by Eq. (4.6). Suppose also that a viscosity law is given in the form $\mu=\mu(T)$, so that a corresponding mean viscosity $\mu_{s}=\mu\left(T_{s}\right)$ can be associated in turn with this mean temperature.

Finally, consider once more the experimentally established properties of the ratio $\bar{\mu} / \sigma$ appearing in the denominator of Eq. (4.7). According to Fig. 5, $\bar{\mu} / \sigma$ is a quantity having the dimensions of viscosity and having moreover a quite definite value at a given station in an adiabatic compressible turbulent boundary layer at constant pressure. This value appears to lie between $\mu_{\infty}$ and $\mu_{\mathrm{w}}$ and to approach the latter as the Reynolds number increases for fixed Mach number. Now the mean viscosity $\mu_{\mathrm{s}}$ corresponding to the mean sublayer temperature $T_{s}$ ought to have very nearly these same properties, not only for flow at constant pressure but for adiabatic flow in general. It is therefore natural to ask, especially after contemplating the numerator of Eq. (4.7), whether or not the quantities $\bar{\mu} / \sigma$ and $\mu_{s}$ might in fact be identical. If so, then

$$
\frac{\vec{\mu}}{\sigma \mu_{\mathrm{w}}}=\frac{\mu_{\mathrm{s}}}{\mu_{\mathrm{w}}}=\frac{\mu\left(T_{\mathrm{s}}\right)}{\mu\left(T_{\mathrm{w}}\right)}=\frac{\rho_{\mathrm{w}}}{\rho_{\infty}} \frac{\bar{C}_{\mathrm{f}}}{C_{\mathrm{f}}} .
$$

The first equality in this expression, the equality $\sigma=\bar{\mu} / \mu_{\mathrm{s}}$, is referred to as the sublayer hypothesis. This relationship evidently provides the third condition needed to define the three scaling functions of the transformation and is the main contribution of this report to the empirical description of the turbulent boundary layer in compressible flow. If 
the sublayer hypothesis is adopted, Eq. (4.7) becomes finally

$$
\operatorname{Re}_{\mathrm{s}}=\rho_{\mathrm{s}} u_{\mathrm{s}} y_{\mathrm{s}} / \mu_{\mathrm{s}}=\text { constant. }
$$

The essence of this argument is the proposal ${ }^{26}$ that the sublayer Reynolds number $\operatorname{Re}_{s}$ may be independent of compressibility when the density and viscosity are evaluated at a suitably defined mean temperature $T_{\mathrm{s}}$. The constant $\mathrm{Re}_{\mathrm{s}}$ in $\mathrm{Eq}$. (4.9), being determined by the properties of the law of the wall for incompressible flow, is conceptually independent of viscosity law, state equation, Mach number, Prandtl number, specific heat ratio, surfacetemperature distribution, heat-transfer rate, etc., in the compressible flow, and may also be taken as independent of Reynolds number and pressure gradient to the same extent as the law of the wall itself. On the other hand, this constant is certainly affected by surface roughness and by mass transfer, and it may possibly be affected by external turbulence level and by changes in geometry such as the introduction of lateral curvature.

\section{Flow of a Perfect Gas}

Given the sublayer hypothesis of Eq. (4.8), the immediate problem becomes the calculation of the sublayer mean temperature $T_{\mathrm{s}}$. For this purpose it is convenient to take the compressible fluid to be a perfect gas having the state equation $p=\rho R T$, and to write from Eq. (4.6),

$$
\frac{1}{T_{\mathrm{s}}}=\frac{R \rho_{\mathrm{s}}}{p}=\frac{R}{p y_{\mathrm{s}}} \int_{0}^{y_{\mathrm{s}}} \rho d y=\frac{1}{y_{\mathrm{s}}} \int_{0}^{y_{\mathrm{s}}} \frac{d y}{T} .
$$

This equation may first be put in the alternative form

$$
T_{\mathrm{s}}=\frac{1}{\bar{y}_{\mathrm{s}}} \int_{0}^{\bar{y}_{\mathrm{s}}} T d \bar{y}=\frac{1}{z_{\mathrm{s}}} \int_{0}^{z_{\mathrm{s}}} T d z,
$$

where $z$ is the independent variable used to represent the mean-velocity profile by way of the law of the wall, Eq. (4.3). The last result may be rewritten once more in terms of the local stagnation temperature

${ }^{26}$ Unfortunately this proposal is simply an invention, and like most inventions is not unique. For example, the sub-layer Reynolds number might equally well have been defined, say, as the definite integral of $f d z$ rather than as the product $f z$. The mean density $\rho_{\mathrm{s}}$ would then be replaced by a mean mass flow, and the transition to a mean temperature and a mean viscosity would become more difficult. Even the latter transition is ambiguous unless it is assumed, as in the text, that mean thermodynamic variables for the sublayer are related by the same laws which apply for corresponding local variables. This assumption, however, requires that the mean temperature be treated as a derived rather than a fundamental quantity. In this respect, as well as in the application to the sublayer and the formation of the mean in terms of an integral determined by the structure of the incomplete equations of motion, the present definition of mean temperature differs from similar but more empirical definitions in the heat-transfer literature.
$T_{0}=T+u^{2} / 2 c_{p}$ and partially evaluated with the aid of Eq. (4.4) to obtain

$$
\frac{T_{\mathrm{s}}}{T_{\mathrm{w}}}=1-\left\langle f^{2}\right\rangle \frac{\gamma-1}{2 \gamma} \frac{\mu_{\mathrm{s}}}{\mu_{\mathrm{w}}} \frac{\tau_{\mathrm{w}}}{p}+\frac{1}{z_{\mathrm{s}}} \int_{0}^{z_{\mathrm{s}}}\left(\frac{T_{0}}{T_{\mathrm{w}}}-1\right) d z,
$$

where by definition

$$
\left\langle f^{2}\right\rangle\left(z_{\mathrm{s}}\right)=\frac{1}{z_{\mathrm{s}}} \int_{0}^{z_{\mathrm{s}}} f^{2} d z=f_{\mathrm{s}}^{2} \frac{\int_{0}^{y_{\mathrm{s}}} \rho u^{2} d y}{u_{\mathrm{s}}^{2} \int_{0}^{y_{\mathrm{s}}} \rho d y}
$$

is the mean value of $f^{2}$ in the sublayer.

\section{The Putative Case $\operatorname{Pr}=1$}

The form of the last term in Eq. (4.12) calls attention to the importance of the special case $T_{0}=T_{\mathrm{w}}=$ constant, and suggests an examination of the consequences of the full Crocco energy integral

$$
c_{p} T+\frac{u^{2}}{2}=c_{p} T_{0}=c_{p} T_{\mathrm{w}}-\frac{q_{\mathrm{w}}}{\tau_{\mathrm{w}}} u,
$$

which is known to be valid for laminar flow with $\operatorname{Pr}=1$ if either the pressure gradient or the heat transfer is zero. On using Eq. (4.14) to evaluate the definite integral in Eq. (4.12), there is obtained

$$
\begin{aligned}
\frac{T_{\mathrm{s}}}{T_{\mathrm{w}}}=\frac{\rho_{\mathrm{w}}}{\rho_{\mathrm{s}}}=1-\langle f\rangle\left(\frac{\mu_{\mathrm{g}}}{\mu_{\mathrm{w}}}\right)^{\frac{1}{2}} \frac{q_{\mathrm{w}}}{\rho_{\mathrm{w}} c_{p} T_{\mathrm{w}}\left(\tau_{\mathrm{w}} / \rho_{\mathrm{w}}\right)^{\frac{1}{2}}} \\
-\left\langle f^{2}\right\rangle \frac{\gamma-1}{2 \gamma} \frac{\mu_{\mathrm{s}}}{\mu_{\mathrm{w}}} \frac{\tau_{\mathrm{w}}}{p},
\end{aligned}
$$

where by definition $q_{\mathrm{w}}=-[k(\partial T / \partial y)]_{\mathrm{w}}, \operatorname{Pr}=c_{p} \mu / k$, and

$$
\langle f\rangle\left(z_{\mathrm{s}}\right)=\frac{1}{z_{\mathrm{s}}} \int_{0}^{z_{\mathrm{s}}} f d z=f_{\mathrm{s}} \frac{\int_{0}^{y_{\mathrm{s}}} \rho u d y}{u_{\mathrm{s}} \int_{0}^{y_{\mathrm{s}}} \rho d y} .
$$

An interesting alternative form of Eq. (4.15) follows when $\tau_{\mathrm{w}} / p$ is replaced by $\gamma M_{\infty}^{2} C_{f} / 2$ and $q_{\mathrm{w}} / \tau_{\mathrm{w}}$ is replaced by $c_{p}\left(T_{\mathrm{w}}-T_{0_{\infty}}\right) / u_{\infty}$, the latter from Eq. (4.14). If $M_{\infty}$ is eliminated from the resulting expression using the definition $T_{0_{\infty}} / T_{\infty}=1+(\gamma-1) M_{\infty}^{2} / 2$, and if $C_{f}$ is eliminated using Eq. (4.8), the result is the simple expression

$$
\frac{T_{\mathrm{s}}}{T_{\mathrm{w}}}=1-\langle f\rangle\left(1-\frac{T_{0_{\infty}}}{T_{\mathrm{w}}}\right)\left(\frac{\bar{C}_{f}}{2}\right)^{\frac{1}{2}}-\left\langle f^{2}\right\rangle\left(\frac{T_{0_{\infty}}}{T_{\mathrm{w}}}-\frac{T_{\infty}}{T_{\mathrm{w}}}\right) \frac{\bar{C}_{f}}{2}
$$

Given the Mach number and the wall-temperature ratio for the compressible flow, therefore, the two formulas (4.17) and

$$
C_{\mathrm{f}}=\frac{T_{\infty}}{T_{w}} \frac{\mu_{\mathrm{w}}}{\mu_{\mathrm{s}}} \bar{C}_{\mathrm{f}}
$$


together provide a parametric relationship-with the viscosity law for $\mu_{\mathrm{s}}=\mu\left(T_{\mathrm{g}}\right)$ as parametric function-expressing the local friction coefficient $C_{\mathrm{f}}$ in terms of $\bar{C}_{\mathrm{f}}$ alone. Once $C_{\mathrm{f}}$ is known, $\operatorname{Re}_{\theta}$ may be found from the law of corresponding stations, Eq. (2.21), and the heat transfer may be computed from the usual formula

$$
C_{\mathrm{h}}=\frac{q_{\mathrm{w}}}{\rho_{\infty} u_{\infty} c_{p}\left(T_{\mathrm{w}}-T_{0_{\infty}}\right)}=\frac{C_{\mathrm{f}}}{2} .
$$

These results, of course, apply only for flows characterized by the Crocco energy integral, Eq. (4.14). Thus the Prandtl number must be unity, ${ }^{27}$ the wall temperature must be constant, and in all probability either the heat transfer or the pressure gradient must be zero, although there is so far no restriction on the viscosity law. Within these limitations, and in particular for the case $d p / d x=0$, the only new empirical information needed is the numerical value of the sublayer Reynolds number $\operatorname{Re}_{s}$ (or of any one of the equivalent quantities $f_{\mathrm{s}}, z_{\mathrm{s}},\langle f\rangle$, or $\left.\left\langle f^{2}\right\rangle\right)$.

\section{The Further Restriction $\mathfrak{u} / T=$ constant}

It will be recalled from Sec. III that the equations of motion for laminar compressible flow could be reduced to incompressible form only for a perfect gas with viscosity proportional to temperature. For turbulent flow the latter restriction, although it may not be necessary, also leads to a particularly simple relationship between the local friction coefficients $C_{\mathrm{f}}$ and $\bar{C}_{\mathrm{f}}$. If $\mu$ is any explicit function of $T$, the sublayer mean temperature $T_{\mathrm{s}}$ can be eliminated from the parametric representation given by Eqs. (4.17) and (4.18). In particular, if $\mu / T=$ constant,

${ }^{27}$ For turbulent flow the validity of the Crocco energy integral Eq. (4.14) - or of any other energy integral in analytical form, for that matter-is a matter for conjecture. The most that can be argued from the linear behavior of $T$ and $u$ near $y=0$ is that the integral Eq. (4.14), if is it valid at all for turbulent flow with heat transfer, can only be valid if the laminar Prandtl number is unity. It follows that calculations based on this integral, which is to say calculations based on Eqs. (4.17) and (4.19), cannot be quantitatively correct for gases such as air, although they may be useful as a means of estimating the effects of compressibility and heat transfer on skin friction.

With regard to Eq. (4.17), it should be remembered that the quantity $T_{8}$ is actually defined, e.g., by Eq. (4.15) or more generally by $\mathrm{Eq}$. (4.10), without reference to conditions in the free stream. On the other hand, Eq. (4.17) has the advantage of being independent of the form of the viscosity law. Furthermore, Eq. (4.17) is readily applied to various special cases of practical interest, including the case of lowspeed heat transfer $\left(M_{\infty}=0\right.$ or $\left.T_{0 \infty} \stackrel{=}{=} T_{\infty}\right)$; the case of adiabatic flow $\left(q_{\mathrm{w}}=0\right.$ or $\left.T_{\mathrm{w}}=T_{000}\right)$; and the case of a very cold wall ( $T_{\mathrm{w}}=0$, say). Finally, Eq. (4.17) expresses the sublayer mean temperature $T_{\mathrm{s}}$ as a linear combination of the three characteristic temperatures $T_{\infty}, T_{0 \infty}$, and $T_{\mathrm{w}}$ and thus bears a strong resemblance to certain empirical formulas that are discussed at greater length in the Appendix. there is obtained

$$
C_{\mathrm{f}}=\frac{\bar{C}_{\mathrm{f}}}{\frac{T_{\mathrm{w}}}{T_{\infty}}-\langle f\rangle\left(\frac{T_{\mathrm{w}}-T_{0_{\infty}}}{T_{\infty}}\right)\left(\frac{\bar{C}_{\mathrm{f}}}{2}\right)^{\frac{1}{2}}-\left\langle f^{2}\right\rangle\left(\frac{T_{0_{\infty}}-T_{\infty}}{T_{\infty}}\right) \frac{\bar{C}_{\mathrm{f}}}{2}},
$$

where corresponding stations are now connected by Eq. (2.21) in the form

$$
C_{\mathrm{f}} \operatorname{Re}_{\theta}=\bar{C}_{\mathrm{f}} \overline{\operatorname{Re}}_{\bar{\theta}} .
$$

For flow at constant pressure, incidentally, the transformation for the streamwise coordinate $x$ can be found from Eq. (4.2), which has so far not been used. In combination with Eqs. (4.1) and (2.19), this equation can be written

$$
\frac{1}{\xi}=\frac{d x}{d \bar{x}}=\frac{1}{\eta} \frac{\bar{\rho} \bar{\mu}}{\rho_{\mathrm{w}} \mu_{\mathrm{w}}} \frac{d \bar{\theta} / \sigma}{d \bar{\theta}} .
$$

But $\sigma$ is equal to $\bar{\mu} / \mu_{\mathrm{s}}$ by the sublayer hypothesis, and $\mu_{\mathrm{s}}$ is an implicit function of $\theta$ through the dependence of both $\overline{\mathrm{Re}}_{\bar{\theta}}$ and $\mu_{\mathrm{w}} / \mu_{\mathrm{s}}$ on $\bar{C}_{\mathrm{f}}$, a dependence given by Fig. 4 in the one case and by the viscosity law and Eq. (4.17), say, in the other. Thus for the special case $T_{0}=$ constant and $\mu / T=$ constant, Eq. (4.22) and the definitions

$$
d \mathrm{Re}_{x}=2 \frac{d \mathrm{Re}_{\theta}}{C_{\mathrm{f}}}, \quad d \overline{\mathrm{Re}}_{\bar{x}}=2 \frac{d \overline{\mathrm{Re}}_{\bar{\theta}}}{\bar{C}_{f}}
$$

together imply

$d \operatorname{Re}_{x}=\left(\frac{T_{\mathrm{s}}}{T_{\infty}}\right)^{2}\left[1-\left(\frac{T_{\mathrm{w}}}{T_{\mathrm{s}}}-1\right) \frac{d \ln \bar{C}_{\mathrm{f}}}{d \ln \overline{\operatorname{Re}}_{\bar{\theta}}}\right] d \overline{\operatorname{Re}}_{\bar{x}}$,

where $\overline{\mathrm{Re}_{\bar{x}}}$ is known empirically as a function of $\overline{\mathrm{Re}}_{\bar{\theta}}$ from a numerical integration of the second of $\mathrm{Eq}$. (4.23). The spirit of this calculation, it should be noted, is typical of most boundary-layer problems, in that $x$ and $\bar{x}$ have to be treated as dependent variables whose evaluation is possible only after the dependence of $\bar{C}_{f}$ on $\overline{\operatorname{Re}}_{\bar{\theta}}$ has been specified in the definition Eq. (4.23).

\section{The Numerical Value of $\mathrm{Re}_{\mathrm{s}}$}

It remains to determine the numerical magnitude of the sublayer Reynolds number $\mathrm{Re}_{s}$. For this purpose the special formulas just obtained are of academic interest only, since these formulas refer for the most part to the flow of a fictitious perfect gas with $\operatorname{Pr}=1$ and $\mu / T=$ constant. Ideally, the best procedure is a direct evaluation of $\mathrm{Re}_{s}$ from the definition (4.9), using measured values for $u(y)$ and $T(y)$. Such measurements would first determine the 


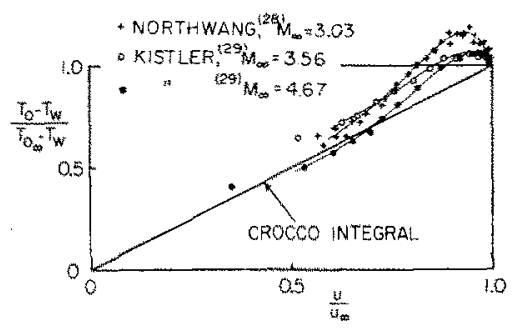

FI. 6. Stagnation-temperature distribution in adiabatic supersonic flow. Data from Refs. 28 and 29.

ratio $\mu_{\mathrm{s}} / \mu_{\mathrm{w}}=\mu / \alpha \mu_{\mathrm{w}}$, either by the methods already employed in Fig. 5 or more generally by a fit to the law of the wall, Eq. (4.4). Given $\mu_{\mathrm{s}}$ and a viscosity law, the implied value of $T_{\mathrm{s}}$ together with the static temperature profile $T(y)$ would then determine $\rho_{\mathrm{s}}$ and $y_{\mathrm{s}}$, the former from the state equation and the latter as the upper limit of integration in Eq. (4.10). With $\rho_{s y} \mu_{s}, y_{s}$, and $u_{s}=u\left(y_{s}\right)$ known, the value of $\operatorname{Re}_{s}=\rho_{\mathrm{s}} u_{\mathrm{s}} y_{\mathrm{s}} / \mu_{\mathrm{s}}$ follows immediately. Moreover, a comparison of values of $R e_{s}$ obtained for various flows by this method would provide a direct and decisive test of the sublayer hypothesis. Unfortunately, the necessary measurements have never been made, so that less direct and hence less decisive methods must be found.

Consider, therefore, the general Eq. (4.12) rewritten in the form

$\frac{T_{\mathrm{w}}}{T_{s}}(1+\epsilon)=1+\left\langle f^{2}\right\rangle \frac{\mu_{s}}{\mu_{\mathrm{w}}} \frac{T_{\mathrm{w}}}{T_{\mathrm{w}}} \frac{\gamma-1}{2} M_{\mathrm{w}}^{2} \frac{C_{f}}{2}$,

where

$$
\epsilon=\left\langle\frac{T_{0}}{T_{\mathrm{w}}}-1\right\rangle=\frac{1}{z_{\mathrm{s}}} \int_{0}^{z_{\mathrm{s}}}\left(\frac{T_{0}}{T_{\mathrm{w}}}-1\right) d z .
$$

Experimentally speaking, the unknown quantities in Eq. (4.25) are the sublayer parameter $\left\langle f^{2}\right\rangle$, which is defined by Eq. (4.13), and the auxiliary parameter $\epsilon$, which represents the effect of variations in stagnation temperature of whatever nature or origin. This parameter $\epsilon$ is presumably zero for adiabatic flow of a fluid with a Prandtl number of unity. If the Prandtl number is not too far from unity, however, the definition (4.26) indicates that $\epsilon$ will be of order $1-r$ for adiabatic flow, where $r$ is the recovery factor. For the purpose of determining $\left\langle f^{2}\right\rangle$ (and hence $\mathrm{Re}_{\mathrm{B}}$ ) from $\mathrm{Eq}$. (4.25), therefore, a relatively rough estimate of $\epsilon$ should be sufficient. This estimate in turn requires a knowledge of an energy integral, i.e., a relationship between temperature and velocity such that $T_{0}(y)$ in Eq. (4.26) can be expressed in terms of $u(y)$ and thus in terms of $f(z)$. The most accurate measurements of $T_{0}(y)$ in adiabatic supersonic flow at con- stant pressure are probably those by Nothwang ${ }^{28}$ and by Kistler, ${ }^{29}$ both of whom measured $T_{0}$ using an unheated hot wire as a resistance thermometer. The measurements in question, shown in Fig. 6, suggest that the observed relationship between velocity and temperature might be satisfactorily approximated-. in the region that is important for the evaluation of $\epsilon$, and only for the purpose of this evaluation-by the straight line ${ }^{30}$

$$
\frac{T_{0}}{T_{\mathrm{w}}}-1=\left(\frac{T_{0_{\infty}}}{T_{\mathrm{w}}}-1\right) \frac{u_{w}}{u_{\infty}}
$$

But this empirical expression for adiabatic llow, with $\operatorname{Pr} \neq 1$, is formally identical with the Croceo energy integral (4.14) for flow with heat transfer, but with $\operatorname{Pr}=1$. It follows that $\epsilon$ can be computed in either case from the formula

$$
\epsilon=\langle f\rangle\left(\frac{T_{0 \infty}}{T_{\mathrm{w}}}-1\right)\left(\frac{\bar{C}_{\mathrm{f}}}{2}\right)^{\frac{1}{2}}
$$

where $\langle f\rangle$ is defined by Eq. (4.16). It also follows that Eq. (4.17) for the mean sublayer temperature $T_{\mathrm{s}}$, although originally derived from the Crocco integral, should also be reasonably accurate for air, at least when the pressure is constant and the flow is nearly adiabatic.

Given Eqs. (4.25) and (4.28), a simple iteration starting with $\epsilon=0$ may be used to find $\langle f\rangle$ and $\left\langle f^{2}\right\rangle$ for the data previously collected in Figs. 1 through 5 . The final result of this iteration is shown in Fig. 7 . The heavy straight line in Fig. $7^{31}$ corresponds to the values $\langle f\rangle=17.2,\left\langle f^{2}\right\rangle=305$; and the light lines illustrate the effect of Reynolds number on $T_{\mathrm{w}} / T_{\mathrm{s}}$ (or on $\vec{\mu} / \sigma \mu_{\mathrm{w}}$ ) at constant Mach number, an effect which was originally cited as the major cause of the scatter in Figs. 2 and 5.

Whether or not the argument for a straight line in Fig. 7 is convincing, one last inference to be drawn from this argument is that any such line must terminate at a finite point, which may be called the

${ }^{28}$ G. J. Nothwang, NACA Technical Note 3721 (1956).

s. A. Kistler, Ballistic Researeh Lab. (Aberdeen) Report 1052 (1958).

${ }^{30}$ The accuracy of this approximation for both low-speed and high-speed flows with heat transfer has to be tested experimentally. It should be emphasized that the object here is to estimate the effect of Prandtil number on skin friction, not to determine the heat transfer. Neither the derivative $(\partial T / \partial y)_{w}$, which is zero for adiabatic flow, nor the integral of $p u\left(T_{0}-T_{\text {ibo }}\right)$ through the boundary layer, which is constant for adiabatic flow, can be correctly obtained from the empirical formula $(4.27)$.

${ }_{31}$ Figure 7 shows the same data as Fig. 3 , except that the ordinate in Fig. 3 is $\mu_{w} / \mu_{a}$ rather than $T_{w} / T_{s}(1+\epsilon)$. Here and elsewhere in this section the sublayer parameters have been computed from the definitions, e.g., Eqs. (4.5), (4.13), or (4.16), using the particular function $f(\xi)$ tabulated in Ref. 25 , but with $f$ reduced by $2 \%$. 
hypersonic limit. The reason is that $C_{\mathbf{f}}$ varies as $1 / M_{\infty}^{2}$ for large $M_{\infty}$, so that $T_{\mathrm{w}} / T_{\mathrm{s}}$ is a bounded function of both Mach number and Reynolds number. From Eq. (4.17) it then follows, for $\left\langle f^{2}\right\rangle=305$ and $\bar{C}_{\mathrm{f}_{\mathrm{max}}}=0.005$, say, that $T_{\mathrm{w}} / T_{\mathrm{s}}$ should have a maximum value of about 6 in adiabatic flow. Furthermore, the product $M_{\infty}^{2} C_{\mathrm{t}}$ is equivalent to $\tau_{\mathrm{w}} / p$, so that for fixed $\bar{C}_{\mathrm{f}}$ the ratio of $\tau_{\mathrm{w}}$ to $p$ (or to $\rho c_{p} T$ ) should remain finite in the limit of large Mach number, despite the fact that $\tau_{\mathrm{w}} / q_{\infty}$ and $p / q_{\infty}$ separately approach zero. Although these conclusions are strictly correct only for the case of a perfect gas with $\mu / T=$ constant and $T_{0}=$ constant, similar conclusions may be expected to hold for real turbulent flows under appropriate conditions.

Finally, the over-all accuracy of the transformation and of the experiments may be tested by reducing the available supersonic data to equivalent incompressible form. Values of $\vec{C}_{\mathrm{f}}$ computed from Eqs. (4.8) and (4.17), using experimental values for $C_{\mathrm{f}}, M_{\infty}, T_{\mathrm{w}} / T_{0_{\infty}}$, the viscosity law, and the sublayer constants, are superimposed in Fig. 8 on the low-speed friction curve of Fig. 4 . In view of the discrepancies that are already present in the raw data as a result of unavoidable irregularities in tunnel conditions, residual effects of tripping devices, and other causes, this final correlation is entirely satisfactory.

\section{The Notion of a Substructure}

The sublayer constants of Sec. IV have now acquired the values

$$
\begin{gathered}
\left\langle f^{2}\right\rangle=305, \quad\langle f\rangle=17.2, \quad f_{\mathrm{s}}=19.8, \quad z_{\mathrm{s}}=430, \\
\operatorname{Re}_{\mathrm{s}}=8500 .
\end{gathered}
$$

At first glance the figure quoted for $\mathrm{Re}_{\mathrm{s}}$ is entirely unprepossessing, because it is many times larger than the Reynolds number of perhaps 150 which is usually taken to be characteristic of the viscous

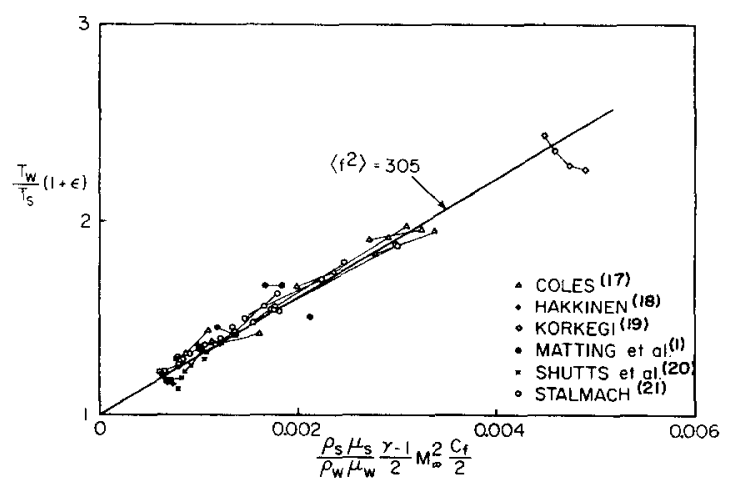

FIG. 7. Indirect evaluation of the substructure Reynolds number Res. Data from Refs. 1, 17-21.

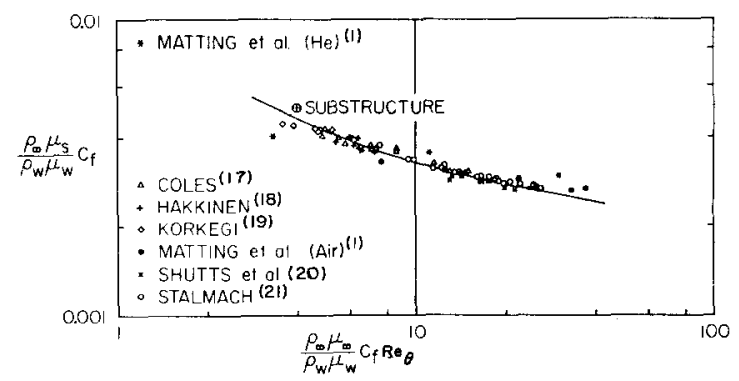

FIG. 8. Friction data in compressible flow according to the transformation. Data from Refs. 1, 17-21.

sublayer in turbulent flow. Equally unprepossessing is the fact that the parameter $\mathrm{Re}_{\mathrm{s}}$, although it is formally defined exclusively in terms of the properties of incompressible flows, seems to play no explicit part in such flows. In other words, $\operatorname{Re}_{\mathrm{s}}$ seems to be important only insofar as the effects of compressibility are concerned, and can in fact be determined only by a study of these effects.

It may be possible to counter both of these objections by proposing a new interpretation for the characteristic Reynolds number $\mathrm{Re}_{\mathrm{s}}$. This interpretation emerges from a study of the available data for low-speed flow, when attention is focused on the inverse transition from turbulent to laminar flow as the Reynolds number decreases under strongly disturbed conditions. ${ }^{32}$ Not only is there a fairly definite critical Reynolds number for this inverse transition, but the limiting turbulent profile near transition seems to be closely described by the law of the wall, Eq. (4.3), terminated not far from the point $\left(f_{\mathrm{s}}, z_{\mathrm{s}}\right)=(19.8,430)$ which now defines the outer edge of the sublayer. The analogy with the critical Reynolds number for flow in a circular pipe is clear, especially since this critical Reynolds number for pipe flow is known to describe not a situation marked primarily by large direct dissipation of energy or by strong damping of velocity fluctuations, but a situation marked by all the essential properties of turbulent shear flow, including the ability to extract energy from the environment-through the action of large eddies having a scale distinct from the dissipation scale - at a rate sufficient to prevent the decay of the turbulence. If this tentative interpretation is adopted for the boundary layer as well, the region in question should probably be given a special name to distinguish it from the ordinary sublayer. The term turbulent substructure is therefore proposed as one which conveys something of the

${ }^{32}$ The study referred to can be found in Appendix $A$ of Rand Report R-403-PR (1962). Except for the one appendix, this Rand Report is essentially the same as the present paper and has the same title. 


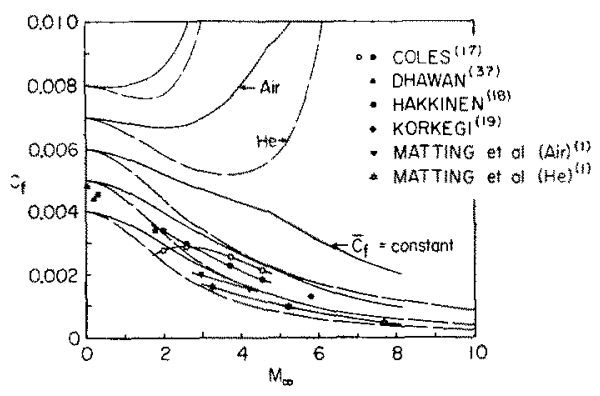

Fig. 9. Maximum local friction coefficients as observed in air and helium (open symbols, natural transition; solid symbols, tripped). Data from Refs. 1, 17-19, 37 .

connection with transition and at the same time indicates the subordinate role played by this region in flow at large Reynolds numbers.

Experimental evidence can be found both for and against the proposition that the substructure parameter $\mathrm{Re}_{\mathrm{s}}$ can be identified with the limiting Reynolds number for turbulent boundary-layer flow. According to the available data, turbulent flow may have been observed at Reynolds numbers $\overline{\operatorname{Re}}_{\bar{q}}=\bar{u}_{\infty} \bar{\theta} / \bar{\nu}$ as small as 290 , whereas the value of $\overline{\mathrm{Re}_{\bar{\theta}}}$ that is characteristic of the substructure flow (with $\bar{u}_{\infty} \bar{\delta} / \bar{\nu}=8500$ ) is 780. However, boundary-layer flows are often classified as turbulent on the basis of indirect evidence, and there is no assurance in some cases that the profiles nearest transition are fully developed in the sense, say, that the intermittency factor is unity near the surface. The main difficulty in the case of boundary-layer flow is that the inherent increase in Reynolds numbers with distance may act to convert a temporary, abnormal response to a strong disturbance into a permanent one. No such difficulty is encountered in the case of pipe flow, for which the critical Reynolds number can be determined with good precision, regardless of initial disturbances, simply by observing the flow far downstream. Preston $^{33}$ has used this property of pipe flow in estimating a minimum value $\overline{\operatorname{Re}}_{\bar{\theta}}=320$ for the boundary layer, but his arguments also rely in part on a defect law that is now known to fail at small Reynolds numbers. In fact, the disappearance of the wake component in both pipe and boundary-layer flow as the Reynolds number decreases almost certainly corresponds to the disappearance of the large eddies that are primarily responsible for the transfer of energy from the mean flow to the turbulence. Perhaps it is also relevant here to mention the value $\widehat{\operatorname{Re}}_{\bar{\theta}}=680$ observed by Dutton ${ }^{34}$ for the asymptotic

${ }^{33}$ J. H. Preston, J. Fluid Mech. 3, 373 (1958). (1955) turbulent boundary layer with suction, a flow in which the wake component is again absent and the survival of the turbulence is again marginal. ${ }^{34}$

Another and less ambiguous approach to the problem of a limiting flow can be made in terms of the maximum value of the local friction coefficient, inasmuch as the properties of any limiting flow should be suitable grist for the mill of the transformation. In several of the previously cited experiments with floating elements, transition was observed to move over the element in response to changes in tunnel pressure or velocity, or in response to changes in the strength of a variable tripping device. The measured values of $C_{f_{\max }}$ are plotted against $M_{\infty}$ in Fig. 9 without regard to the corresponding values of $\mathrm{Re}_{\hat{\xi}}$, which were often not measured and might in any case be difficult to guarantee. Also shown are some curves of constant $\bar{C}_{\mathrm{f}}$ computed for air and helium at wind-tunnel conditions ${ }^{35}$ with the aid of the general transformation, using for the sublayer parameters the numerical values just quoted. The figure indicates that values of $\bar{C}_{\mathrm{f}}$ larger than 0.0048 are not in fact associated with any of these floating-element experiments, ${ }^{36}$ although strenuous efforts were made in most cases to stimulate early transition to turbulent flow. The available data are therefore consistent with a plausible upper bound on $\bar{C}_{\mathrm{f}}$ supplied by the value $2 / f_{\mathrm{s}}^{2}=0.0051$ for the hypothetical substructure flow with $\overline{\mathrm{Re}}_{\bar{\theta}}=780$.

\section{APPENDIX. THE RELATED ANALYTICAL LITERATURE}

\section{The Effective-Temperature Hypothesis}

Because the analysis presented in this paper has elements in common with several previous contributions to the literature of turbulent compressible

${ }^{35}$ The term wind-tunnel conditions means that the stream stagnation temperature for air has been taken to be $550^{\circ} \mathbf{R}$ for $M_{\infty}<4.7$, and the stream static temperature to be $100^{\circ} \mathrm{R}$ for $M_{\infty}>4.7$. The increase in $C_{\mathrm{f}} / \bar{C}_{\mathrm{g}}$ with increasing $\mathrm{Mach}$ number, when $\bar{C}_{\hat{t}}$ is large, corresponds qualitatively to the change in sign of the factor multiplying $M^{2}$ in the denominator of Eq. (A18) of the Appendix. For the special conditions represented by the latter formula it then follows that $T_{\mathrm{a}} / T_{\infty}$ is less than unity wheneyer $\bar{C}_{\mathrm{f}}$ exceeds the value $2 /\left\langle f^{2}\right\rangle \stackrel{\infty}{=}$ 0.0066 . Since this condition cannot be met in an adiabatic flow in which $T$ decreases monotonically from $T_{\mathrm{w}}$ at the wall to $T_{\infty}$ in the free stream, a definite upper bound in the neighborhood of $\bar{C}_{\mathrm{q}}=0.0066$ is seen to be inherent in the formalism of the substructure concept itself.

${ }_{36}$ The correction for gap effect (the inclusion of the gap area as part of the element area) has not been made for Dhawan's data, ${ }^{37}$ as it amounts to a decrease in $\bar{C}_{f}$ of some $15 \%$. Figure 9 also confirms a conclusion which the author reached and reported at the time of his own experiments; in Ref. 17, this conclusion is that the fence tripping device sometimes had the peculiar effect of inhibiting rather than stimulating transition. (1951). 
flow, a brief review of the relevant literature is in order. This review considers, without attempting to be exhaustive, (1) the concept of a mean or effective temperature as a basis for estimating the effect of compressibility on turbulent skin friction; (2) the concept of a laminar film, or sublayer, whose properties control the friction and especially the heat transfer; and (3) previous work on transformations designed to reduce the equations of mean motion partly or wholly to incompressible form.

The main assumptions of the effective-temperature hypothesis are that a friction law is given for incompressible flow in the form $\bar{C}_{f}=\bar{C}_{f}(\overline{\mathrm{Re}})$, and that this same law is valid for compressible flow provided only that the density and viscosity appearing in the friction law (as distinguished from the density and viscosity appearing in the definitions of $C_{f}$ and $\mathrm{Re}$ ) are evaluated at a suitably chosen mean or effective temperature $T_{\mathrm{m}}$.

In other. words, $\bar{C}_{\mathrm{f}}$ and $\overline{\mathrm{Re}}$ are to be replaced in the friction law by

and by

$$
\bar{C}_{\mathrm{f}}=\frac{2 \tau_{\mathrm{w}}}{\rho_{\mathrm{m}} u_{\infty}^{2}}=\frac{\rho_{\infty}}{\rho_{\mathrm{m}}} \frac{2 \tau_{\mathrm{w}}}{\rho_{\infty} u_{\infty}^{2}}=\frac{\rho_{\infty}}{\rho_{\mathrm{m}}} C_{\mathrm{f}}
$$

$$
\widehat{\mathrm{Re}}=\frac{\rho_{\mathrm{m}} u_{\infty} L}{\mu_{\mathrm{m}}}=\frac{\rho_{\mathrm{m}}}{\rho_{\infty}} \frac{\mu_{\infty}}{\mu_{\mathrm{m}}} \frac{\rho_{\infty} u_{\infty} L}{\mu_{\infty}}=\frac{\rho_{\mathrm{m}}}{\rho_{\infty}} \frac{\mu_{\infty}}{\mu_{\mathrm{m}}} \mathrm{Re},
$$

respectively. These two relationships, insofar as they provide rules for computing $C_{\mathrm{f}}$ and $\operatorname{Re}$ when $\bar{C}_{\mathrm{f}}$ and $\overline{\mathrm{Re}}$ are given, are equivalent to a transformation. The proper choice of the reference length $L$ in Eq. (A2), however, is obvious only for certain special cases of laminar flow. For example, if the pressure is constant and the fluid is a perfect gas with viscosity proportional to temperature, the combinations $C_{\mathrm{f}}\left(\mathrm{Re}_{x}\right)^{\frac{1}{2}}$ and $C_{\mathrm{f}} \operatorname{Re}_{\theta}$ are known to be independent of Reynolds number, Mach number, Prandtl number, and heat-transfer rate. It follows that the equations $\bar{C}_{\mathrm{f}}=C_{\mathrm{f}} T_{\mathrm{m}} / T_{\infty}$ and $\overline{\mathbf{R e}}_{L}=$ $\operatorname{Re}_{L}\left(T_{\infty} / T_{\mathrm{m}}\right)^{2}$ are consistent for an arbitrary choice of $T_{\mathrm{m}}$ if $L=x$, but are inconsistent if $L=\theta$. In the later case it is evidently necessary to put, instead of Eq. (A2),

$$
\overline{\operatorname{Re}}_{\theta}=\frac{\rho_{\infty} u_{\infty} \theta}{\mu_{\mathrm{m}}}=\frac{\mu_{\infty}}{\mu_{\mathrm{m}}} \frac{\rho_{\infty} u_{\infty} \theta}{\mu_{\infty}}=\frac{\mu_{\infty}}{\mu_{\mathrm{m}}} \operatorname{Re}_{\theta},
$$

or perhaps

$$
\overline{\operatorname{Re}}_{\theta}=\frac{\rho_{\mathrm{m}} u_{\infty} \theta}{\mu_{\infty}}=\frac{\rho_{\mathrm{m}}}{\rho_{\infty}} \frac{\rho_{\infty} u_{\infty} \theta}{\mu_{\infty}}=\frac{\rho_{\mathrm{m}}}{\rho_{\infty}} \operatorname{Re}_{\theta} .
$$

Thus, if $L=\theta$, the viscosity or the density, but not both, should be evaluated at the mean temperature $T_{\mathrm{m}}$. Alternatively, Eq. (A3) can be derived by assuming that Eq. (A2) is correct when $L=x$ and that mean and local friction coefficients should transform according to the same rule.

The quantitative formulation of the effectivetemperature concept ${ }^{38}$ begins with a combination of Eqs. (A1) and (A2) in the form

$$
\bar{C}_{\mathrm{f}}\left(\overline{\operatorname{Re}}_{x}\right)^{\frac{1}{2}}=\left(\frac{\rho_{\infty} \mu_{\infty}}{\rho_{\mathrm{m}} \mu_{\mathrm{m}}}\right)^{\frac{1}{2}} C_{f}\left(\operatorname{Re}_{x}\right)^{\frac{1}{2}}
$$

which is appropriate for laminar flow with similarity when the pressure is constant. If the fluid is a perfect gas but the viscosity is not proportional to temperature, the product $C_{\mathrm{f}}\left(\mathrm{Re}_{x}\right)^{\frac{1}{2}}$ will depend on Prandtl number, Mach number, and heat-transfer rate. This dependence can first be determined with the aid of exact solutions of the laminar boundary-layer equations, whereupon Eq. (A5) can be used to find an appropriate value of $T_{\mathrm{m}}$, hopefully as a linear combination of $T_{\infty}, T_{\mathrm{w}}$, and $T_{0_{\infty}}$ (or $T_{\mathrm{a}_{w}}$, the adiabatic wall temperature). This formula for $T_{\mathrm{m}}$, in conjunction with the relationships (A1) and (A2) with $L=x$, then serves as an interpolation and extrapolation device for estimating skin friction and heat transfer when exact solutions are not available.

The argument given here for laminar flow was first outlined by von Kármán ${ }^{39}$ in his Volta Congress paper of 1935. Von Kármán also suggested a similar interpolation device for turbulent flow and proposed as a first approximation for $T_{\mathrm{m}}$ the wall temperature $T_{\mathrm{w}}$. The qualitative accuracy of this intuitive suggestion has been well established by a gradual accumulation since 1935 of exact solutions of the laminar equations on the one hand and of experimental data for turbulent flow on the other. An almost direct line of descent from von Kármán's formulation to the one in use today can be traced through papers by Rubesin and Johnson, ${ }^{40}$ Young and Janssen, ${ }^{41}$ and Eckert. ${ }^{22,42}$ Experimental contributions to the turbulent problem have also been made by Tucker, ${ }^{43}$ Sommer and Short, ${ }^{44}$ and others. Eckert, in particular, has acquired a kind of propri-

${ }^{38}$ The real origins of this concept are to be found in early work on heat transfer in enclosed channels. For such problems the idea of a bulk or mixing-cup temperature arises quite naturally in any heat-balance calculation. This review, however, is restricted to the concept of mean temperature as it has developed in the context of the boundary-layer equations for compressible flow.

${ }_{39} \mathrm{~T}$. von Kármán, in Proceedings of the Fifth Volta Congress (Reale Accademia d'Italia, Rome, 1936).

${ }^{40} \mathrm{M}$. W. Rubesin, and $\mathrm{H}$. Johnson, Trans. ASME 71, $383(1949)$.

${ }_{41}$ G. B. W. Young, and E. Janssen, Rand Corporation Report P-214 (1951).

${ }_{42}$ E. R. G. Eckert, Wright Air Development Center Technical Report 59-624 (1960).

${ }_{43}$ M. Tucker, NACA Technical Note 2337 (1951).

${ }_{44} \mathrm{~S}$. C. Sommer and B. Short, NACA Technical Note 3391 (1955). 
etary interest in the reference-temperature method by virtue of his participation in applications of this method to laminar problems marked by chemical changes such as dissociation and by variations in surface pressure and temperature. As a result, his 1954 formula for the reference temperature $T_{\mathrm{m}}$,

$$
T_{\mathrm{m}}=0.50\left(T_{\mathrm{w}}+T_{\infty}\right)+0.22\left(T_{\mathrm{a} w}-T_{\infty}\right),
$$

is frequently quoted, as is an equivalent formula for reference enthalpy. At least two writers (Rott, ${ }^{45}$ Burggraf ${ }^{46}$ ) have derived formulas similar to Eq. (A6) by assuming that the characteristic temperature for turbulent flow is the temperature at the edge of the sublayer (but see also the comments following Eq. (A13) and following Eqs. (A15) through (A17) of this appendix). In these two papers, however, the main object is to determine not skin friction but heat transfer in terms of skin friction, and the main emphasis is therefore on the question of an energy integral for turbulent flow. A formula similar to Eq. (A6) has also been derived by Monaghan ${ }^{47}$ using a mean representation of the Crocco energy integral.

In most of this work the independent variable has been the Reynolds number $\mathrm{Re}_{x}$, for reasons that have little relevance for turbulent flow. One obvious difficulty is that there is no generally accepted definition for $\mathrm{Re}_{x}$ in a turbulent boundary layer. Thus Eckert's formula, Eq. (A6), requires $\overline{\mathrm{Re}}_{x} / \mathrm{Re}_{x}=\left(T_{\infty} / T_{\mathrm{m}}\right)^{2}$ to be a rapidly decreasing function of Mach number [i.e., $T_{\mathrm{m}} / T_{\infty}=1+$ $0.72 r(\gamma-1) M_{\infty}^{2} / 2$ for adiabatic flow], and there is no assurance that turbulent compressible flows cannot be observed at values of $M_{\infty}$ and $\mathrm{Re}_{x}$ such that $\overline{R e}_{x}$ is small enough to be off scale from the experimental point of view. If such cases occur, extrapolation of a particular analytical formula for $\bar{C}_{\mathrm{f}}\left(\overline{\mathrm{Re}}_{x}\right)$ to low Reynolds numbers in order to generate values for $\bar{C}_{\mathrm{f}}$ and $C_{\mathrm{f}}$ is a questionable procedure at best.

It is therefore unfortunate that much of the conceptual simplicity of the effective-temperature method is lost in working with local quantities, as for example when Eq. (A1) is combined with the awkward Eq. (A3) to obtain

$$
\bar{C}_{\mathrm{f}} \overline{\operatorname{Re}}_{\theta}=\left(\rho_{\infty} \mu_{\infty} / \rho_{\mathrm{m}} \mu_{\mathrm{m}}\right) C_{\mathrm{f}} \operatorname{Re}_{\theta} .
$$

This expression bears a superficial resemblance to

\footnotetext{
${ }^{45}$ N. Rott, Ramo-Wooldridge Corporation GMRD Report GM-TR-211 (1957).

${ }_{46}$ O. R. Burggraf, Lockheed Aircraft Corporation Report LSMD-895080 (1961).

${ }_{47}$ R. J. Monaghan, in Proceedings of the Fifth International Aeronautical Conference, edited by R. J. Turino (Institute of Aeronautical Science, New York, 1955), p. 277.
}

the law of corresponding stations, Eq. (2.21) of the text. The law of corresponding stations, however, is part of a genuine mapping of physical flow variables, while Eq. (A7) is probably best described as a plausible but somewhat arbitrary relationship between certain dimensionless parameters of the problem. The same distinction can be made between Eqs. (4.17) and (A6) for the characteristic temperatures $T_{\mathrm{s}}$ and $T_{\mathrm{m}}$, respectively, and between Eqs. (4.18) and (A1) for the local friction coefficients. In fact, for the case of viscosity proportional to temperature, the latter two equations become formally identical when $T_{\mathrm{s}}$ is identified with $T_{\mathrm{m}}$, and Eckert's formula (A6) for adiabatic flow can then be plotted in Fig. 5 as the straight line $T_{\mathrm{m}} / T_{\mathrm{w}}=$ $T_{\infty} \bar{C}_{\mathrm{f}} / T_{\mathrm{w}} C_{\mathrm{f}}=0.72+0.28 T_{\infty} / T_{\mathrm{w}}$.

\section{The Laminar-Film Hypothesis}

A second empirical approach to the problem of the turbulent boundary layer is based on the film or sublayer hypothesis. For incompressible flow, for example, the assumption is that the mean-velocity profile can be adequately represented by a combination of a linear profile $\bar{u}=\bar{\tau}_{w} \bar{y} / \bar{\mu}$ near the wall and by, say, a power law $\bar{u} / \bar{u}_{\infty}=(\bar{y} / \bar{\delta})^{1 / n}$ elsewhere in the boundary layer. The two curves intersect at the edge of the sublayer, where $\bar{u}$ and $\bar{y}$ have the values $\bar{u}_{\mathrm{f}}$ and $\bar{y}_{\mathrm{f}}$, respectively. Once the ratios $\bar{u}_{i} / \bar{u}_{\infty}$ and $\bar{y}_{i} / \bar{\delta}$ have been evaluated in terms of $\bar{C}_{\mathrm{f}}$ and $\overline{\mathrm{Re}}_{\bar{\delta}}$, it is clear that one further condition relating $\bar{u}_{\mathrm{f}}$ to $\bar{y}_{\mathrm{f}}$ will determine a local friction law in the form $\bar{C}_{f}=\bar{C}_{\mathrm{f}}\left(\overline{\operatorname{Re}}_{\bar{\delta}}\right)$. The condition in question may be obtained by assuming a constant sublayer Reynolds number $\operatorname{Re}_{f}=\bar{u}_{\mathrm{f}} \bar{y}_{\mathrm{f}} / \bar{\nu}$, or alternatively by assuming a constant ratio of laminar to turbulent shearing stress at the edge of the sublayer, the stresses being evaluated with the aid of a suitable mixing-length formula. For the case of a power-law profile outside a linear sublayer these two assumptions can be shown to be completely interchangeable.

This formulation of the film hypothesis was begun independently by Prandtl $^{48}$ and Taylor ${ }^{49}$ in connection with the problem of turbulent heat transfer. It was later carried essentially to completion by Prandtl, ${ }^{50}$ who derived a suitable sublayer condition from the Blasius local friction law rather than vice versa. The formulation described here is due to Donaldson, ${ }^{51,52}$ who also extended his analysis to

${ }^{48}$ L. Prandtl, Physik. Z. 11, 1072 (1910).

${ }^{49}$ G. I. Taylor, Report and Memoranda 272 (1916).

${ }^{50}$ L. Prandtl, Physik. Z. 29, 487 (1928).

51 C. duPont Donaldson, NACA Technical Note 2692 (1952).

${ }_{62}$ In the opinion of the author Donaldson's paper deserves a prominent place in any review of the analytical literature 
the case of high-speed flow by assuming in effect that the film Reynolds number, redefined as $\operatorname{Re}_{\mathrm{f}}=$ $\rho_{i} u_{i} y_{i} / \mu_{i}$, should be unaffected by compressibility or heat transfer. The new variables $\rho_{f}$ and $\mu_{f}$ at the edge of the sublayer appear both in $\operatorname{Re}_{\mathrm{f}}$ and in the formula $\tau_{\mathrm{w}}=\mu_{\mathrm{f}} u_{\mathrm{i}} / y_{\mathrm{t}}$, and they can be determined once a viscosity law and an energy integral have been specified.

Among the relationships derived explicitly or implicitly in Donaldson's original paper are

$$
\begin{gathered}
C_{\mathrm{f}} \operatorname{Re}_{\theta}=2 \frac{\theta}{\delta} \frac{T_{\mathrm{f}}}{T_{\infty}}\left(\frac{u_{\mathrm{f}}}{u_{\infty}}\right)^{1-n}, \\
C_{\mathrm{f}}=\frac{2}{\operatorname{Re}_{\mathrm{f}}} \frac{T_{\infty}}{T_{\mathrm{f}}}\left(\frac{u_{\mathrm{f}}}{u_{\infty}}\right)^{2}, \\
\frac{T_{\mathrm{f}}}{T_{\infty}}=1+\frac{\gamma-1}{2} M_{\infty}^{2}\left[1-\left(\frac{u_{\mathrm{f}}}{u_{\infty}}\right)^{2}\right],
\end{gathered}
$$

where it has been assumed for the sake of simplicity that $T_{0}$ is constant and that $\mu$ is proportional to $T$. The first of these equations is obtained by eliminating $y_{\mathrm{f}}$ between the linear and power-law profiles at their intersection, ${ }^{53}$ the second by eliminating $y_{\mathrm{f}}$ in favor of $\mathrm{Re}_{\mathrm{f}}$ in the linear profile, and the third by putting $u=u_{\mathrm{f}}$ and $T=T_{\mathrm{f}}$ in the energy integral $C_{p} T+\frac{1}{2} u^{2}=C_{p} T_{0}=$ constant.

If now $u_{t} / u_{\infty}$ is eliminated between Eqs. (A8) and (A9) on the one hand and between Eqs. (A9) and (A10) on the other, a local friction law for $C_{f}\left(\mathrm{Re}_{\theta}\right)$ is obtained in parametric form as

$C_{\mathrm{f}}=2 \operatorname{Re}_{\mathrm{f}}^{(1-n) /(1+n)}\left(\frac{T_{\infty}}{T_{\mathrm{f}}}\right)^{(n-3) /(n+1)}\left(\frac{\delta}{\theta} \operatorname{Re}_{\theta}\right)^{-2 /(n+1)}$,

$$
\frac{T_{\mathrm{w}}}{T_{\mathrm{f}}}=1+\frac{\operatorname{Re}_{\mathrm{f}}}{2} \frac{\gamma-1}{2} M_{\infty}^{2} C_{\mathrm{f}} .
$$

For $M_{\infty}=0$ and $n=7, \mathrm{Eq}$. (A11) becomes the Blasius law $C_{\mathrm{f}}=0.045 \mathrm{Re}_{b}^{-\frac{1}{4}}$, in which the numerical coefficient 0.045 implies the value $\operatorname{Re}_{f}=158$ noted by Donaldson.

It is worth noting that Eq. (A11), which is the same as Eq. (17) of Donaldson, ${ }^{51}$ can be rewritten for a general viscosity law in the terminology of the effective-temperature concept; thus

of the compressible turbulent boundary layer. The reason is that this paper has stood almost alone, pending the first applications of coordinate transformations to turbulent flow, in recognizing the increase in the relative thickness of the sublayer at large Mach numbers as the dominant effect of compressibility.

${ }_{53}$ Tables of the ratio $\theta / \delta$, which depends on $M_{\infty}$, on $T_{\mathrm{w}} / T_{\mathrm{co}}$ and on the exponent $n$ in the power-law profile, have been published by several authors; e.g., Tucker (Ref. 43), and Persh and Lee [J. Persh and R. Lee, Navord Report 4282 (1956)], for the more general density-velocity relationship given by the Crocco energy integral.

$$
\frac{2 \tau_{\mathrm{w}}}{\rho_{\mathrm{f}} u_{\infty}^{2}}=2 \operatorname{Re}_{\mathrm{f}}^{(1-n) /(1+n)}\left(\frac{\rho_{\mathrm{f}} u_{\infty} \delta}{\mu_{\mathrm{f}}}\right)^{-2 /(n+1)}
$$

Within the limitations of the power-law approach, therefore, the local friction law in the form $C_{\mathrm{f}}=$ $C_{f}\left(\mathrm{Re}_{\delta}\right)$ is found to be unaffected by compressibility or heat transfer if the density and viscosity are evaluated at a film temperature $T_{\mathrm{f}}$, which is defined for the case of constant stagnation temperature by Eq. (A12). Consequently, one effect of the special assumptions of the film hypothesis, as compared with the much less specific assumptions of the effective-temperature hypothesis, is to provide an explicit formula (showing a weak dependence on Reynolds number) for an effective temperature $T_{\mathrm{f}}$. The film analysis, however, does not appear to be equivalent to a transformation, since no rules are provided for the identification of corresponding stations.

Finally, the close resemblance of the formula (A12) to the corresponding case of Eq. (4.25),

$$
\frac{T_{\mathrm{w}}}{T_{\mathrm{s}}}=1+\frac{\left\langle f^{2}\right\rangle}{2} \frac{\gamma-1}{2} M_{\infty}^{2} C_{\mathrm{f}},
$$

also deserves a comment. The linear sublayer profile is a rudimentary form of the law of the wall, and the condition $\mathrm{Re}_{\mathrm{f}}=$ constant is a general consequence of the latter law, at least for the case of incompressible flow. Donaldson's intuitive and indirect extension of this condition to the compressible case (in terms of the ratio of shearing stresses rather than the Reynolds number $\mathrm{Re}_{i}$, and without benefit of any real similarity law for the mean-velocity profile) sets a precedent which the present author is pleased to follow. However, the arguments at the beginning of Sec. IV proceed by way of the full law of the wall, the transformation, and the presentation of experimental data in Fig. 5; and the most important element common to the two approaches is the emphasis on conditions within and near the edge of the sublayer. Moreover, Donaldson apparently did not notice-perhaps because he did not actually write out Eq. (A12) or perhaps because his analysis neither suggested nor required such a result-that the effective temperature ratio $T_{\mathrm{f}} / T_{\mathrm{w}}$ in this equation is in fact a function only of $\gamma$ and $\tau_{\mathrm{w}} / p$ and is thus formally independent of conditions in the outer flow, including the exponent $n$ in the power-law representation of the mean-velocity profile.

An analysis not unlike that by Donaldson has also been described by Spence, ${ }^{54}$ who combined a

54 D. A. Spence, Royal Aircraft Establishment, Farnborough, Report Aero 2631 (1959). 
power-law outer profile with the law of the wall near the surface, the two curves intersecting (more properly, osculating) at a point in the logarithmic region of the latter law. On matching the velocity gradients as well as the velocities at this point, he obtained a power law for $\bar{C}_{\mathrm{f}}\left(\overline{\mathrm{Re}}_{\delta}\right)$. Spence's treatment of the compressible case included the substitution of $\rho_{\mathrm{m}}$ for $\bar{\rho}$ and $\mu_{\mathrm{m}}$ for $\bar{\mu}$, with $\rho_{\mathrm{m}}$ and $\mu_{\mathrm{m}}$ evaluated at Eckert's mean temperature. He also introduced a partial transformation by substituting $\eta=\int_{0}^{\nu}\left(\rho / \rho_{\mathrm{m}}\right) d y$ for $\bar{y}$. The resulting local friction law differs only slightly from Donaldson's formula (A13). Spence also examined a limited amount of experimental data in transformed coordinates. His most significant contribution to the problem of the turbulent boundary layer, however, is probably his ingenious derivation of a power-law shearing-stress profile for use in the integration of the energy equation.

\section{Transformations}

The prior art in transformation of the turbulentboundary-layer equations begins with papers by Dorodnitsyn $^{10}$ and Van Le, ${ }^{55}$ both of whom considered only the momentum-integral equation of von Kármán. The first attempt to transform the differential equations of mean motion for a turbulent boundary layer was made by Mager $^{56}$ in an analysis that is restricted to the case of adiabatic flow of a perfect gas with $\operatorname{Pr}=1$ and $\mu / T=$ constant. Mager's three main assumptions are (1) that streamlines should be transformed into streamlines by the transformation; (2) that laminar and turbulent shearing stresses should be transformed by the same rules (in Mager's paper this assumption is expressed by a remarkable rationalization that has sometimes been repeated by later writers); and (3) that the transformation should reduce to the identity transformation when both flows are incompressible.

The third of these three assumptions accounts for the fact that the concept of effective temperature is frequently invoked in connection with the transformation. Most writers on the subject have considered one or more of the relationships (here written in the present notation, with viscosity taken as proportional to temperature),

$$
\begin{array}{r}
\frac{C_{\mathrm{f}}}{\bar{C}_{\mathrm{f}}}=\frac{\bar{\rho}}{\rho_{\infty}}=\frac{\mu_{\infty}}{\bar{\mu}}, \\
\frac{\operatorname{Re}_{\theta}}{\overline{\overline{R e}_{\bar{\theta}}}}=\frac{\rho_{\infty}}{\bar{\rho}}=\frac{\bar{\mu}}{\mu_{\infty}},
\end{array}
$$

${ }_{56}$ N. Van Le, J. Aeron. Sci. 20, 583 (1953).

${ }^{56}$ A. Mager, J. Aeron. Sci. 25, 305 (1958).

$$
\frac{\operatorname{Re}_{x}}{\operatorname{Re}_{\bar{x}}}=\frac{\rho_{\infty}}{\bar{\rho}} \frac{\bar{\mu}}{\mu_{\infty}}=\left(\frac{\rho_{\infty}}{\bar{\rho}}\right)^{2}=\left(\frac{\bar{\mu}}{\mu_{\infty}}\right)^{2} .
$$

The only distinction ordinarily made between these equations and the corresponding Eqs. (A1) through (A3) of the effective-temperature analysis is that the reference quantities $\bar{\rho}$ and $\bar{\mu}$ represent fluid properties in an incompressible flow that is related to the compressible one by the transformation, whereas the reference quantities $\rho_{\mathrm{m}}$ and $\mu_{\mathrm{m}}$ refer to fluid properties at some intrinsic reference state for the compressible flow itself. If the transformation is required to reduce to the identity transformation when both flows are incompressible, this becomes a distinction without a difference, and the choice of a reference state for the transformation is equivalent to an application of the effective-temperature method.

Thus Mager took $\bar{\rho}$ and $\bar{\mu}$ to be the isentropic stagnation values for the compressible flow and used Eqs. (A15) and (A17) and a suitable low-speed friction law to compute $C_{\mathrm{f}} / \bar{C}_{\mathrm{f}}=\mu_{\infty} / \mu_{0_{\infty}}$ for fixed $C_{\mathrm{f}}\left(\mathrm{Re}_{x}\right)^{\frac{1}{2}}$. In this calculation, which is very like that of von Kármán in Ref. 39, Mager assumed a power law for $\mu(T)$ in place of the relationship $\mu / T=$ constant which is appropriate to his transformation. Burggraf in Ref. 46 later modified Mager's result by evaluating $\bar{\rho}$ and $\bar{\mu}$ at the edge of the sublayer, i.e., at the intersection of the linear and logarithmic representations for the meanvelocity profile, without commenting on the fact that the fluid properties in the incompressible flow must then depend on $x$. Culick and Hill, ${ }^{57}$ making a point of $\mathrm{Eq}$. (A16) but not specifically of the law of corresponding stations, determined $\bar{\mu} / \mu_{\infty}$ from Eq. (A15) by using experimental values of $C_{\mathrm{f}} / \bar{C}_{\mathrm{f}}$ for fixed $C_{\mathrm{f}} \operatorname{Re}_{\theta}$. They also concluded, independently of Mager, that $\bar{\rho}$ and $\bar{\mu}$ should be evaluated at the isentropic stagnation condition (but note the contradictory evidence in Fig. 5). Similar comments apply to a number of other analyses, ${ }^{58}$ most of which are applications of the transformation technique to various practical problems.

It may be appropriate to conclude by commenting briefly on what might be called the unfinished business of the transformation. For the purposes of this discussion it is instructive to compare the transformation to a bilingual dictionary, which is to be used to translate statements about compressible

${ }^{57}$ F. E. C. Culick and J. Hill, J. Aeron. Sci. 25, 259 (1958). ${ }_{68}$ In the present analysis the equations corresponding to Eqs. (A15) through (A17) are Eqs. (2.17), (2.20), and (4.24); the solution proposed for the problem of a reference temperature is the sublayer hypothesis; and the $x$ dependence of the reference properties is assigned to the parameter $\sigma(x)=$ $\bar{\mu} / \mu_{\mathrm{B}}(x)$ rather than to the parameter $\bar{\mu}$ alone. 
flows into corresponding statements about associated incompressible flows, and vice versa. The construction of such a dictionary must ensure that the sense of certain fundamental statements, particularly physical laws for the conservation of mass and momentum, together with supplementary ideas about boundedness and observability of physical quantities, is correctly translated. For more specialized subjects, however, the vocabulary of the transformation has to be enlarged. By definition, any material used for this purpose must itself be capable of translation, although it may be empirical and heuristic in nature. Typical examples of such special vocabularies are those provided by (1) the assumption of Newtonian friction for laminar flow, and (2) the sublayer hypothesis for the turbulent boundary layer on a smooth wall.

A number of problems not yet touched upon in this paper may now be classified in terms of this metaphor. The turbulent wake and the turbulent boundary layer on a rough surface, for example, require their own special vocabularies. In both cases the viscosity is an artificial parameter, and some of the relationships derived here--beginning with the law of corresponding stations in the case of the boundary layer-have to be reconsidered. Inasmuch as an adequate description of the mean properties of low-speed flow is available in both cases, and inasmuch as the effects of compressibility on drag for rough surfaces are also known, neither of these problems seems to be really formidable.

Certain other problems, however, require some preliminary work in composition before the question of translation can even be raised. Prominent among these problems are the effects of mass transfer and of pressure gradient in compressible turbulent flow. In the one case the methods of this report seem to require first a satisfactory generalization of the law of the wall for low-speed flow with mass transfer, and then a corresponding generalization of the sublayer hypothesis. In the other case, that of flow with pressure gradient, there is no real difficulty with the sublayer hypothesis, or with the derivation of formulas like

$$
\frac{C_{\mathrm{f}}}{\bar{C}_{\mathrm{f}}}=\frac{1}{1+\left(1-\left\langle f^{2}\right\rangle \frac{1}{2} \bar{C}_{\mathrm{f}}\right) \frac{1}{2}(\gamma-1) M_{\infty}^{2}},
$$

which follow from this hypothesis for special cases such as adiabatic flow with $T_{0}=$ constant and $\mu / T=$ constant. Instead, the difficulty is with the condition connecting the two quantities $M_{\infty}$ and $\bar{C}_{f}$ on the right-hand side of this formula when both quantities are variable. This difficulty can be illus- trated by recalling the situation in laminar flow, for which it was shown in Sec. III that the condition $d p / d x=0$ implies $\eta / \sigma=u_{\infty} / \bar{u}_{\infty}=$ constant, whereas a special argument based on the assumption that $\tau$ should transform like $\mu(\partial u / \partial y)$ throughout the flow was necessary in order to extend this result-in the form $\eta^{2} / \sigma^{2}=T_{\infty} / \bar{T}$, or $M_{\infty}=\bar{u}_{\infty} /(\gamma R \bar{T})^{\frac{1}{2}}$, with the auxiliary restrictions $T_{0}=$ constant and $\mu / T=$ constant-to the case $d p / d x \neq 0$. For turbulent flow the condition $d p / d x=0$ still implies $\eta / \sigma=$ constant, but the argument used for the case $d p / d x \neq 0$ no longer holds. Consequently the transformation cannot yet be applied with confidence to turbulent flow in a pressure gradient, regardless of the methods used to overcome the additional difficulty of relating the incompressible variables $\bar{u}_{\infty}$ and $\bar{C}_{f}$ in the associated low-speed flow. Moreover, the status of the conditions $T_{0}=$ constant and $\mu / T=$ constant for flows with pressure gradient is at best uncertain.

Finally, the problem of heat transfer is typical of several problems that must always lie outside the scope of any transformation incorporating the idea of observability. The reason is that equations of state, energy, or concentration involve concepts existing only in one language and not in the other, concepts which are inherently incapable of being translated. At the same time, such concepts enter into the description of physical quantities in the compressible flow and introduce complications in both the direct and the inverse transformations. As long as the common practice of honoring the energy equation in the breach is continued, therefore, the most elegant results of any transformation are likely to be the least realistic ones, as in the present paper, and no new contributions to the subject of heat transfer in turbulent flow can be expected. Quite possibly more attention should be paid, both experimentally and analytically, to the development of similarity laws for static and stagnation temperature. It has been emphasized throughout this report that the turbulent shearing stress can be readily inferred from a knowledge of the velocity and pressure fields; in fact, a vehicle for this calculation in the case of compressible flow has been provided in Eq. (3.11). At least in principle, the turbulent heat transfer can be inferred in a similar way from an empirical knowledge of the velocity, pressure, and temperature fields, and the relationship between the shearing stress $\tau$ and the heat transfer $q$ in turbulent flow might thus be made experimentally accessible without recourse to difficult hot-wire techniques for the direct measurements of these quantities. 